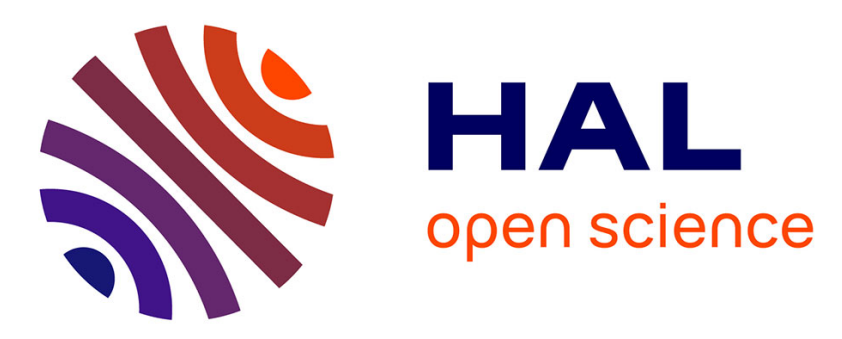

\title{
Identification of NLR-associated amyloid signaling motifs in bacterial genomes.
}

\author{
Witold Dyrka, Virginie Coustou, Asen Daskalov, Alons Lends, Thierry \\ Bardin, Mélanie Berbon, Brice Kauffmann, Corinne Blancard, Bénédicte
}

Salin, Antoine Loquet, et al.

\section{To cite this version:}

Witold Dyrka, Virginie Coustou, Asen Daskalov, Alons Lends, Thierry Bardin, et al.. Identification of NLR-associated amyloid signaling motifs in bacterial genomes.. Journal of Molecular Biology, 2020, 432, pp.6005 - 6027. 10.1016/j.jmb.2020.10.004 . hal-02989877

\section{HAL Id: hal-02989877 https://hal.science/hal-02989877}

Submitted on 16 Dec 2020

HAL is a multi-disciplinary open access archive for the deposit and dissemination of scientific research documents, whether they are published or not. The documents may come from teaching and research institutions in France or abroad, or from public or private research centers.
L'archive ouverte pluridisciplinaire HAL, est destinée au dépôt et à la diffusion de documents scientifiques de niveau recherche, publiés ou non, émanant des établissements d'enseignement et de recherche français ou étrangers, des laboratoires publics ou privés. 


\section{Journal Pre-proofs}

Research Article

Identification of NLR-associated amyloid signaling motifs in bacterial genomes

Witold Dyrka, Virginie Coustou, Asen Daskalov, Alons Lends, Thierry Bardin, Mélanie Berbon, Brice Kauffmann, Corinne Blancard, Bénédicte Salin, Antoine Loquet, Sven J. Saupe

PII: S0022-2836(20)30580-5

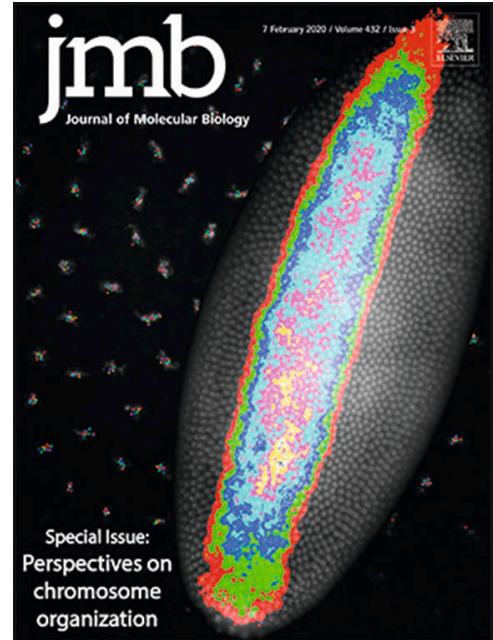

DOI: https://doi.org/10.1016/j.jmb.2020.10.004

Reference: YJMBI 66669

To appear in: Journal of Molecular Biology

Received Date: 24 April 2020

Revised Date: 5 October 2020

Accepted Date:

5 October 2020

Please cite this article as: W. Dyrka, V. Coustou, A. Daskalov, A. Lends, T. Bardin, M. Berbon, B. Kauffmann, C. Blancard, B. Salin, A. Loquet, S.J. Saupe, Identification of NLR-associated amyloid signaling motifs in bacterial genomes, Journal of Molecular Biology (2020), doi: https://doi.org/10.1016/j.jmb.2020.10.004

This is a PDF file of an article that has undergone enhancements after acceptance, such as the addition of a cover page and metadata, and formatting for readability, but it is not yet the definitive version of record. This version will undergo additional copyediting, typesetting and review before it is published in its final form, but we are providing this version to give early visibility of the article. Please note that, during the production process, errors may be discovered which could affect the content, and all legal disclaimers that apply to the journal pertain.

\section{Elsevier Ltd}




\section{Identification of NLR-associated amyloid signaling motifs in bacterial genomes}

Witold Dyrka ${ }^{2}$, Virginie Coustou ${ }^{1}$, Asen Daskalov ${ }^{4}$, Alons Lends ${ }^{4}$, Thierry Bardin ${ }^{1}$, Mélanie Berbon ${ }^{3}$, Brice Kauffmann ${ }^{5}$, Corinne Blancard ${ }^{3}$, Bénédicte Salin ${ }^{3}$, Antoine Loquet ${ }^{4}$ and Sven J. Saupe ${ }^{1}$

${ }^{1}$ Non-self recognition in fungi, Institut de Biochimie et de Génétique Cellulaire, UMR 5095 CNRS, Université de Bordeaux, 1 rue Camille Saint Saëns, 33077 Bordeaux CEDEX, France, sven.saupe@ibgc.cnrs.fr, corresponding author

${ }^{2}$ Politechnika Wrocławska, Wydział Podstawowych Problemów Techniki Katedra Inżynierii Biomedycznej

Wybrzeże Wyspiańskiego 27, 50-370 Wrocław, Poland

${ }^{3}$ Institut de Biochimie et de Génétique Cellulaire, UMR 5095 CNRS, Université de Bordeaux, 1 rue Camille Saint Saëns, 33077 Bordeaux CEDEX, France

${ }^{4}$ Institute of Chemistry \& Biology of Membranes \& Nanoobjects, UMR5248 CBMN, IECB, CNRS, Université de Bordeaux, Allee Geoffroy Saint-Hilaire, 33607 Pessac, France

${ }^{5}$ IECB, UMS 3033, US 001, CNRS, Université de Bordeaux, 2 rue Robert Escarpit, 33607, Pessac, France 


\begin{abstract}
In filamentous fungi, amyloid signaling sequences allow Nod-like receptors (NLRs) to activate downstream cell-death inducing proteins with HeLo and HeLo-like (HELL) domains and amyloid RHIM and RHIM-related motifs control immune defense pathways in mammals and flies. Herein, we show bioinformatically that analogous amyloid signaling motifs exist in bacteria. These short motifs are found at the N-terminus of NLRs and at the C-terminus of proteins with a domain we term BELL. The corresponding NLR and BELL proteins are encoded by adjacent genes. We identify 10 families of such bacterial amyloid signaling sequences (BASS), one of which (BASS3) is homologous to RHIM and a fungal amyloid motif termed PP. BASS motifs occur nearly exclusively in bacteria forming multicellular structures (mainly in Actinobacteria and Cyanobacteria). We analyze experimentally a subset of seven of these motifs (from the most common BASS1 family and the RHIM-related BASS3 family) and find that these sequences form fibrils in vitro. Using a fungal in vivo model, we show that all tested BASSmotifs form prions and that the NLR-side motifs seed prion-formation of the corresponding BELL-side motif. We find that BASS3 motifs show partial prion cross-seeding with mammalian RHIM and fungal PP-motifs and that proline mutations on key positions of the BASS3 core motif, conserved in RHIM and PP-motifs, abolish prion formation. This work expands the paradigm of prion amyloid signaling to multicellular prokaryotes and suggests a long-term evolutionary conservation of these motifs from bacteria, to fungi and animals.
\end{abstract}

\title{
Keywords
}

Signal transduction, programmed cell death, actinobacteria, cyanobacteria, multicellularity 


\section{Introduction}

NLRs are intracellular receptors controlling innate immunity and host-symbiont interactions, both in plants and animals $[1,2]$. NLR proteins have a typical tripartite architecture with an N-terminal effector domain, a central (NACHT or NB-ARC) nucleotide binding and oligomerization domain and a C-terminal leucine-rich repeat (LRR) domain. Filamentous fungi also display large and diverse repertoires of up to several hundreds NLR-related genes per genome [3]. These fungal NLR homologs however display WD40, ANK or TPR repeats as ligand recognition domains instead of LRRs found in most plant and animals NLRs [3,4]. Fungal NLRs were found to control programmed cell death associated with non-self recognition in several fungal species [5-10]. These proteins are considered the fungal counterparts of plant and animal NLRs [3]. Remarkably some fungal NLRs employ an amyloid signaling mechanism to engage cell death inducing effector proteins [11]. These NLRs display a short 20-25 amino acid long N-terminal amyloid forming motif upstream of the NACHT (or NB-ARC) domain while their cognate effector protein displays a similar motif C-terminally. The amyloid fold of the activated NLR receptor serves as a structural template to convert the homologous region in the effector protein to a similar amyloid fold $[7,12,13]$. This signaling mechanism based on the prion principle (self-propagation of protein polymers) is also operating in several immune signaling cascades in mammals, albeit with a different underlying structural basis [14]. In fungi, NLR and their cognate regulated effector proteins are typically encoded by adjacent genes and thus form functional gene pairs or clusters $[13,15,16]$. Several classes of fungal amyloid signaling motifs have been described. HRAM motifs (for $\underline{H E T}$-s Related Amyloid Motifs) (Pfam PF11558) were originally identified in the [Hets] prion of Podospora anserina and have been subject to in-depth functional and structural characterization $[7,15,17,18]$. HRAMs form a $\beta$-solenoid amyloid fold comprising 21 amino acid long pseudo-repeats, with one repeat copy on the NLR and two copies on the effector protein [19]. Another family of signaling amyloids is defined by the sigma motif, involved in the propagation of the $\sigma$ cytoplasmic infectious element of Nectria haematococca [13, 20] (Pfam PF17046). Finally, the PP-motif (for pseudo-palindrome) was described in Chaetomium globosum and displays a sequence similarity with the mammalian RHIM amyloid sequence scaffolding the RIP1K/RIP3K necrosome [16, 21, 22]. RHIM and RHIM-related motifs were also identified in viruses and Drosophila [23, 24]. The similarity between the metazoan RHIM and fungal PP-motifs raised the possibility of an ancient evolutionary origin of this mechanism of cell death-inducing amyloid signaling [16, 25]. The relation between RHIM and PP is further supported by the observation that RHIM and PP-amyloids partially cross-seed in vivo [26].

Several types of downstream effector protein domains activated by NLR-mediated amyloid signaling were described $[3,13]$ but the most common are part of a family of membrane targeting proteins including the HeLo, HELL (Helo-like) and SesA domains (Pfam PF14479, PF17111 and PF17707 respectively). The HeLo domain forms an $\alpha$-helical bundle with an $\mathrm{N}$-terminal hydrophobic helix and functions as a membrane targeting pore-forming domain $[27,28]$. The fungal HeLo/Helo-like/SesA domain family shows homology with the $\mathrm{N}$-terminal helical cell-death execution domain of the MLKL protein controlling mammalian necroptosis and the RPW8 and Rx-N CC-domains regulating plant immune cell death $[16,29]$. The homology between the membrane-targeting domains suggests a common evolutionary origin for these defense-related programmed cell death processes in plants, animals and fungi. The plant Rx_N and the HET-S HeLo domain share a common mechanism of membrane targeting based on the membrane insertion of a hydrophobic $\mathrm{N}$-terminal $\alpha$-helix [27, 30]. Other amyloid-controlled effector domains in fungi are predicted to carry enzymatic activity, in 
particular the SesB $\alpha / \beta$ hydrolase domain and the PNP-UDP phosphorylase domain $[13,16]$. Noteworthy is the observation that fungal NLRs are found in two distinct domain architectures, either as two-component gene clusters involving amyloid signaling or more frequently in an "all-in-one" architecture with the effector/NOD/repeat domains encoded as single polypeptide $[3,13]$.

Several bacterial proteins have received a RHIM amyloid motif annotation in the Pfam or InterPro databases $[22,31]$. We have analyzed the corresponding protein sequences and found that the region annotated as RHIM occurs in a similar genomic context as fungal amyloid signaling motifs. The motif occurs at the N-terminus of a NLR-like protein and at the C-terminus of a putative effector protein encoded by an adjacent gene. Here, we systematically explore in a genome mining approach the occurrence of putative amyloid signaling sequences in bacterial and archaeal genomes and identify ten families of bacterial amyloid signaling sequences (named here BASS1 to 10). We find these motifs mainly in multicellular bacteria in particular in Actinobacteria and Cyanobacteria. We test representatives of the BASS1 and BASS3 families for fibril formation in vitro and prion propagation in the Podospora anserina fungal model. We propose that NLR-associated BASS motifs are analogous to the amyloid prion motifs identified in fungi and that this signaling mechanism is shared by filamentous fungi and filamentous bacteria.

\section{Results}

\section{RHIM-like motifs in Bacteria}

In the Pfam database, the majority of the sequences with a RHIM annotation are from Metazoan or Metazoan viruses [21, 23-25, 32]. A few hits (24/286) however occur in Bacteria. We examined these bacterial RHIM-annotated proteins and found that ten of them are homologous proteins with the RHIM annotation occurring C-terminally, downstream of 100 amino acid long predicted $\alpha$-helical domain that we termed BELL (for $\underline{b}$ acterial domain analogous to HELL, based on shared features with the fungal HeLo-related domains, see below). In the actinobacterium strain Saccharothrix sp. ALI-22-I, the gene adjacent to the gene encoding the BELL/RHIM protein (ONI86675.1) encodes a protein with NLR architecture (NB-ARC domain and TPR repeats) (ONI86674.1). Remarkably, the N-terminal region of this NB-ARC/TPR protein shows sequence homology to the C-terminal RHIM-annotated region of the BELL-domain protein (Fig. $1 \mathrm{~A}$ and B). The ONI86675.1/ ONI86674.1 bacterial gene pair from Saccharothrix therefore displays the same features as effector/NLR gene pairs described in filamentous fungi $[7,13]$. This similarity prompted us to analyze further BELL domain proteins in bacterial genomes.

\section{BELL-domain occurs in multicellular Bacteria and Archaea}

The predicted globular BELL domain of the ONI86675.1 protein from Saccharothrix was used as query in HMMER searches to recover homologous proteins. We found that the BELL-domain occurs mainly in 120-140 amino acid long proteins and in more rare instances as $\mathrm{N}$-terminal domain of proteins with a NLR domain architecture (as previously described for HeLo, HeLo-like and SesA 
domains) [3]. An alignment of BELL domain proteins from phylogenetically diverse prokaryotes including a sequence from an Archaea (Methanothrix soehngenii) is presented Figure 1C. The Nterminal region of the domain was predicted to correspond to an hydrophobic $\alpha$-helix and a HMMsignature of the domain shows frequent occurrence of a negatively charged residue in position 2 or 3 , a feature that is common to fungal HeLo, HeLL domains, mammalian MLKL and plant RPW8 and Rx_N domains (Fig.1D, E and F) [16, 33]. Using contact prediction maps based on evolutionary co-variance, the Bell domain of Saccharothrix sp. ALI-22-I ONI86675.1 was modelled as a five-helix bundle (Fig.1 F). Secondary structure and fold prediction for the different proteins presented in the alignment in Fig. $1 \mathrm{C}$ resulted in five-helix bundles for all proteins although in some cases with different topologies (Fig. S1).

We analyzed the phylogenetic distribution of the BELL domain in prokaryotic genomes using the genome-based phylogeny developed by Parks et al. which has been shown to be more accurate than the NCBI taxonomy [34]. The domain shows a heterogeneous phylogenetic distribution (Table 1, Table2, Table S1). It is most frequent in the Cyanobacteriota, Actinobacteriota and Chloroflexota (present is respectively 33,7 and $4 \%$ of the sequenced genomes) but very rare or absent in other bacterial phyla. Within these phyla, the domain is specifically present in genera containing multicellular species (described in Bergey's Manual of Systematic Bacteriology) (Table S1). In the Actinobacteria class, the domain is frequent in families encompassing filamentous species (the domain occurs in $51 \%$ of Streptomycetaceae, $26 \%$ of the Micromonosporaceae, $46 \%$ of the Pseudonocardiaceae, $33 \%$ of the Streptosporangiaceae, $22 \%$ of the Frankiaceae and $2 \%$ of the Corynebacteriaceae) but absent in unicellulars such as the Bifidobacteriaceae and Propionibacteriaceae (Table 2, Table S1). The same is true at higher phylogenetic resolution, within the Corynebacteriaceae family for instance, the domain is highly represented only in the Nocardia and Dietzia genera encompassing filamentous species (present in $35 \%$ and $21 \%$ of the genomes respectively ) (Table S1). In the Cyanobacteriota, the domain is highly represented in the Nostocaceae family (found in $67 \%$ of the genomes) and present in multicellular genera like Nostoc, Calothrix, Microcystis or Fischerella but absent in the Cyanobiaceae family encompassing unicellular genera like Prochlorococcus and Synechococcus (Table 2, Table S1) [35]. In the Chloroflexota, the domain is specific to the Chloroflexus genus (present in $66 \%$ of the genomes) encompassing filamentous species like Chloroflexus auranticus and Chloroflexus aggregans. Similarly, in Archaea, the BELL domain is found exclusively in the Methanosarcinales order, in particular in the genus Methanosarcina comprising multicellular species forming cell aggregates like Methanosarcina acetivorans (Table S1).

The phylogenetic distribution of the BELL domain is mirrored by the distribution of NLR-like architecture proteins (here a NB-ARC or NACHT domain associated with TPR or WD repeats) (Table 1, Table S1). The NLR-like domain architecture is frequent in phylogenetic groups in which the BELL domain is represented (for instance in Actinobacteriota and Cyanobacteriota) but rare or absent in groups lacking BELL domains (for instance Proteobacteria or Firmicutes) (Table 1). The same is true at higher phylogenetic resolution (Table 2, Table S1). For instance, in Cyanobacteriota, the NLR-like architecture is not found in Cyanobiaceae but is prevalent in Nostocaceae. Similarly, in Archaea, in Methanosarcina, the NLR-like architecture is abundant but rare or absent in other genera. The phylogenetic distributions of NLR-like architectures and BELL domain do not fully overlap though, as for instance the BELL domain is not found in the phylum Myxococcota where NLR-like architectures are present. Conversely, the genus Janthinobacterium contains many BELL domains but lacks NLR-like 
architecture proteins (Table S1). Specific occurrence in Cyanobacteria and Actinobacteria of NLR-type architectures (NB-ARC/NACHT with TPR/WD repeats) was reported previously $[4,36,37]$.

Overall, the BELL domain was found in roughly $1 \%$ of the analyzed bacterial genomes (1237 of $113324)$ and $10 \%$ of the archaeal genomes (94/1183). The domain appears to be typical of Bacteria and Archaea with a multicellular organization either in filamentous or aggregate forming species. In addition, the phylogenetic distribution of the BELL domain proteins is largely mirrored by the phylogenetic distribution of proteins with NLR-like architectures.

\section{Prediction of amyloid signaling sequence motifs in bacterial genomes}

Amyloid signaling motifs in fungi occur in adjacent gene pairs [13]. Based on the observed BELLRHIM/NLR-like gene pairs (Fig. 1A), we hypothesized that an amyloid signaling mechanism akin to the one described in filamentous fungi might also operate in some bacterial species. To identify potential amyloid signaling motifs in bacteria we extended a genome mining strategy that we have previously developed to identify amyloid signaling motifs in fungi $[13,15,16]$. We screened for sequence motifs common to C-termini of BELL-domain proteins and N-termini of NLR-like proteins, which are encoded by adjacent (or closely linked) genes. Specifically, we generated a data set of 1816 non-redundant Ctermini of prokaryotic BELL-domain proteins, searched for conserved gapless motifs using MEME and identified 117 motifs (a general flowchart of the bioinformatics procedure is given Fig. S2). For each of the conserved motifs, we created a profile HMM (pHMM) signature generalizing the motif as described in detail in the Methods section. Next, we identified all NB-ARC and NACHT domain proteins encoded by genes in the genomic vicinity (within $5 \mathrm{kbp}$ ) of the genes encoding our set of BELL-domain proteins. Then, we analyzed N-termini of of these NLR-like proteins for occurrences of the pHMM-signatures of BELL-associated C-terminal motifs. We found that half of the motifs identified at least one gene pair encoding a BELL-domain protein and a NACHT/NB-ARC protein. To be more stringent in our analyses, we focused further investigations only on motifs that identified at least 5 non-redundant gene pairs which reduced our set to 29 motifs (comprising up to 75 non-redundant gene pairs per motif) (Table S2). The motifs were clustered based on the overlap between the matched gene pairs (Fig. S3). When at least half of the gene pairs identified for a given motif were also matched by another motif, the two motifs were considered related and were grouped in the same motif cluster. The procedure led to identification of nine clusters (some of which comprised in fact a single motif) (Fig. S3). Table S3 gives a list and an alignment of all the motif sequences grouped by cluster. In each cluster, we selected a representative motif on the basis of the number of underlying sequences (Fig. 2). The motifs families defined by each cluster were termed BASS families (for bacterial amyloid signaling sequence) (Fig. 2 , Fig. S3). As expected, we recovered the already identified RHIM-related motif represented by 19 gene pairs (BASS3). We also carried out an inverse screen in which we looked for conserved gapless motifs in $\mathrm{N}$-terminal sequences of the NB-ARC/NACHT protein set, generated pHMM signatures for the extracted motifs, and searched for their occurrences in C-termini of the BELL proteins (Fig. S2). We retained 12 motifs identifying at least five non-redundant gene pairs and clustered these motifs within the previously defined clusters. While eleven grouped to one of the nine previously identified families (Fig. S3), one novel motif did not, and is thus defining an additional family, we term BASS10 (Fig.2).

We complemented the search with an alternative procedure consisting of the local pairwise alignment of adjacent BELL C-termini and NLR N-termini. Highly conserved pairs of a length of at least 
15 amino acids were found in 283 gene pairs including 44 pairs not matched previously with the BASS1 to 10 motifs. In this number were two pairs from archaeal Methanosarcinales species (Table S2).

We analyzed the distance and the relative orientation between the BELL encoding gene and the adjacent NLR-encoding gene in our set of 346 matching gene pairs (Table S2). Genes were collinear in $91.3 \%$ of the cases. In most collinear gene pairs, the distance between the genes was very small, in $57 \%$ of the cases the distance between the two ORFs was 10 bp or less and in $42 \%$ of the cases the two ORFs overlapped. In one extreme case, in Spirosome oryzae, the BELL domain encoding gene and the NLR-encoding gene overlapped by $57 \mathrm{bp}$. Gene overlaps are not uncommon in prokaryotes, roughly $20 \%$ of the collinear gene pairs overlap, [38]. It is likely that the BELL and NLR-encoding genes pairs with short intergenic distances or gene overlaps are transcribed as polycistronic messenger RNAs reflecting the possible functional link and/or co-regulation between the genes in the pair.

\section{Paired BASS motifs occur in multicellular species}

As expected from the distribution of BELL-domains and NLRs, the vast majority of BASS-motifs pairs occur in Actinobacteria and Cyanobacteria (Table S1) with the filamentous genus Streptomyces dominating the data set with 105 pairs (Table S2). Some motifs appear specific to Actinobacteria (BASS 1, 2) others to Cyanobacteria (BASS 7, 8, 9, 10), others occur in both classes (BASS 3, 4, 5, 6), (Table S2). All actinobacterial hits occur in filamentous species with the possible exception of Arthrobacter sp. Rue61a. No direct information on the growth morphology for this specific strain could be recovered but the corresponding genus is described as unicellular. The hits in Cyanobacteria correspond essentially to multicellular filamentous species (Nostoc, Pseudoanabaena, Calothrix, Aulosira, Tolothrix, Leptolyngbya genera). Some species with hits (Microcystis aeruginosa and Crocosphaera watsonii) are described as unicellular (non-filamentous) Cyanobacteria but are also found in multicellular aggregates. One hit was found in Cyanothece sp. PCC 7424 which is a unicellular strain reported to form large amounts of mucilage, as well as in Chamaesiphon minutus PCC 6605 a unicellular species forming epiphytic colonies on aquatic plants. Cyanothece, Microcystis, Crocosphaera and Chamaesiphon are however distinct from the main group of unicellular Cyanobacteria (Prochlorococcus, Synechococcus) in terms of phylogenetic position and abundance of secondary metabolism clusters and homologs of AmiC involved in multicellular growth [35]. The remaining gene pairs occur in Archaea, Bacteriodetes and Chloroflexi, in multicellular species, which are either filamentous (like the Chloroflexi Ktedonobacter racemifer) or existing as cellular aggregates (like the Archaea Methanosarcinales species). We conclude that matching BASS-motif pairs occur with a wide phylogenetic distribution but in the vast majority of cases in multicellular strains and genera.

\section{Sequence analysis of BASS motifs}

BASS motifs were typically around 25 amino acid in length (Fig. 2). All motifs have a predicted propensity for $\beta$-sheet formation (of note is the fact that this criterion was not used for their identification). The sequence logos of the motifs show conservation of $G, N$ and $Q$ residues as well as charged residues and conserved patterns of hydrophobic residues. In comparison to the total of Uniprot entries, the motifs are enriched for Q, N, G, V and I and depleted in K, P and L (Table S4). Kajava 
and co-workers have reported a similar bias in amino acid composition of $\beta$-helical proteins with enrichment in $\mathrm{N}, \mathrm{V}$ and $\mathrm{G}$ and depletion in $\mathrm{P}$ and L [39]. Fungal amyloid motifs of the HRAM, sigma and PP family share a similar bias in composition, residue conservation and length [13] (Table S4).

Algorithms predicting amyloid propensity derived from the analysis of pathological amyloids generally perform poorly when run on fungal functional amyloid motifs [40]. In contrast, the ArchCandy program predicting propensity to form $\beta$-arch structures shows good performance for pathological amyloids but also in the case of fungal functional amyloid motifs [41]. We thus evaluated the amyloid propensity of BASS motif containing proteins with ArchCandy and found that their amyloid propensity scores were comparable to those calculated for a selection of validated fungal amyloid motifs and human RHIM-motifs (Fig. S4) (BASS1 and BASS3 scored below the recommended stringent score threshold (0.575) but this was also the case for an HRAM5 fungal prion motif [15]). In each case, the high scoring region matched the position of the identified BASS motif. Based on this analysis, the identified bacterial motifs are predicted to have amyloid forming propensity (note again that they were not selected on this criterion).

The fungal HRAM motifs exemplified by the HET-s prion-forming domain present a two-fold pseudorepeat of the motif in the effector protein $[7,42]$. The two pseudorepeats are separated by a variable flexible glycine and proline -rich loop of 6-15 amino acids in length $[15,17]$. We find that some BASS motifs also occur as pseudorepeats. In proteins pairs of different Streptomyces species, BASS1 and 6 motifs are present in the BELL-domain protein as two (or three) pseudorepeats separated by a variable proline-rich region and as a single repeat in the corresponding NLR (Fig. S5). These motifs thus are similar in this respect to fungal HRAM motifs.

Fungal amyloid signaling motifs can be associated to other effector domains not related to the HeLo-family (HeLo, Helo-like, sesA) but equally encoded by NLR/effector gene pairs $[3,13,16]$. These domains include $\alpha / \beta$-hydrolase and PNP-UDP phosphorylase domains. We wondered therefore whether BASS motifs could also be associated to domains distinct from BELL. Thus, we analyzed the occurrence of identified BASS motifs in gene pairs encoding a NLR and a second protein, for which the motif is found in the $\mathrm{C}$-terminal region next to a domain distinct from BELL. At least four such situations were identified with BASS motifs associated to the following domains : a $\alpha / \beta$-hydrolase domain, the TIR2 domain, the CHAT metacaspase domain and a guanylate cyclase domain (Fig. S6). All identified examples were found in multicellular species. Bacterial genomes contain genes in which the same effector domains are found in an "all-in-one" association, where the effector domain is directly associated to the NOD and repeat domains of the NLR-like protein, as describe previously for fungal NLRs [13]. While some of these domains have rather general functions, it is of interest to note that all these domains ( $\alpha / \beta$-hydrolase, TIR2, CHAT and guanylate cyclase) are to various extends documented to be involved in immune, programmed cell death and biological conflict pathways $[16,37,43-46]$ including in prokaryotes [47].

\section{BASS1 and BASS3 motifs form fibrils in vitro}

Next, for a subset of those motifs, we analyzed in vitro fibril formation. We chose to express a 4 motif sequences one corresponding to the most populated family (BASS 1) from the model species Streptomyces coelicolor A3(2) and the three others BASS3 (RHIM-like motifs) form Actinobacteria 
species Streptomyces atratus and Nocardia fusca and form the Cyanobacteria species Nostoc punctiforme (Table 3, Fig. S7). The three selected BASS3 sequences show less than $50 \%$ identity between species (Fig. S7). To avoid potential difficulties arising from recombinant expression of small peptides, two constructs were expressed for each sequences (except for Nostoc punctiforme ACC79696.1, a short construct limited to approximatively the $30 \mathrm{C}$-terminal residues and a larger constructs encompassing part of the BELL domain (Table 3). All polypeptides were produced in E. coli as inclusion bodies and purified under denaturing conditions in the presence of $6 \mathrm{M} \mathrm{GuHCl}$. Upon dilution of the denaturant, all constructs led to the formation of fibrillar aggregates, either as dispersed fibrils or as laterally associated large bundles. Fibril width was in the range of 5-7 $\mathrm{nm}$ comparable to the previously identified fungal signaling motifs (Fig. 3A), [7, 16, 48]. We further analyzed the in vitro fibrils by ThT-binding (Fig. 3B). Both BASS1 and BASS3-fibrils induced ThT-fluorescence although the signal for BASS3 was low. HET-s(218-289) represents a precedent as an amyloid inducing only very low ThT-fluorescence [48].

For the Streptomyces coelicolor A3(2) BASS1 motif (CAB66307.1, residue 38 to 139) we further assessed fibril structure by X-ray fiber diffraction and solid state NMR. In unoriented BASS1 fibrils, we observed a strong ring at about $4.7 \AA$, and a weaker ring around $10 \AA$ (Fig. $3 C$ ), this pattern being characteristic for a cross- $\beta$ structure corresponding to the inter-strand and inter-sheet spacing respectively, typically observed in amyloid fibrils [49]. Next, we produced a BASS1 sample isotopically and uniformly ${ }^{13} \mathrm{C}$ labeled to carry out solid-state NMR analysis. Cross-polarization ${ }^{13} \mathrm{C}$ experiment (Fig. 3D) revealed a well-resolved spectrum, implying a high structural order at the local level [50]. In line with the X-ray diffraction analysis, solid-state NMR ${ }^{13} \mathrm{C}$ chemical shifts of BASS1 indicate a protein conformation rich in $\beta$-sheet secondary structure, illustrated with a high field effect of the carbonyl region indicative of $\beta$-sheet structure [51]. Taken together, these analyses show that this BASS-motif forms $\beta$-sheet-rich amyloid fibrils, with NMR features comparable to amyloid fibrils of the fungal HET$s(218-289)$ prion $[42,52]$.

\section{BASS1 and BASS3 motifs behave as prion-forming domains when expressed in Podospora}

Fungal amyloid motifs behave as prion-forming domains $[7,16]$. In order to determine whether the BASS motifs could form prions, we expressed selected BASS motifs in $P$. anserina, a model used to analyze prion propagation of both homologous and heterologous amyloid signaling motifs $[7,16,53]$. $P$. anserina is a valuable alternate model to yeast which was also extensively used to document prion properties of heterologous sequences. In $P$. anserina prion propagation and transmission are easy to monitor because of the syncytial structure of the mycelium and because strains spontaneously fuse and mix their cytoplasmic content when confronted [54].. We expressed both the BELL-associated BASS and the corresponding NLR-associated BASS (except for N. fusca proteins for which only the BELLassociated sequence was studied) (Table 3). The seven different BASS motifs were fused with either GFP (in N-terminus) or RFP (in C-terminus) and expressed under a strong constitutive promotor in $P$. anserina (Fig. 4, Fig. S7, Table 3). All fusion proteins displayed bistability, initially showing a diffuse cytoplasmic fluorescence that could convert over time to discrete cytoplasmic dots as previously described for fungal prion-forming domains (Table 4, Fig. 4) [7, 16, 53, 55, 56]. By analogy with fungal prions, we denote the diffuse state $\left[b^{*}\right]$ and the aggregated state $[b]$. Transition from the diffuse $\left[b^{*}\right]$ to the aggregated [b] state could occur either spontaneously or be induced by cytoplasmic contact 
with a strain expressing the fusion protein in [b] state (Fig.5A). We conclude that all tested BASS motifs (both from the NLR and from the Bell-side) direct infectious propagation of the aggregated state and thus behave as prion-forming domains in this fungal model.

It was shown that the globular domains attached to fungal prion domains inhibit prionformation $[16,27]$. To verify if this is also the case for the bacterial BELL-domain, we analyzed prionformation rates the previously tested BASS1 and BASS3 motif now in the full-length protein context. We found that rate formation of the dot state was strongly reduced (Table S5) as compared to the BASS-domain alone constructs (Table 4). Even after long culture periods the rate of dot-formation rates with full length BELL proteins remains in the $2-6 \%$ range while it is typically close to $100 \%$ with the BASS-motif alone constructs. It thus appears, has in the case of the fungal HeLo-domain proteins that presence of the BELL-domain reduces prion-formation rates.

\section{NLR-side BASS induce prion conversion of BELL-side BASS}

If BASS motifs function as fungal amyloid signaling motifs, it is expected that the NLR-side BASS is able to convert the matching BELL-side BASS to the prion state and BELL and NLR-side motifs should co-aggregate [7]. To test whether BASS motif pairs co-localize in aggregates, we co-expressed each motif pair (differentially tagged with GFP and RFP) in the same fungal strain. We found that all tested pairs co-localize in dots although in some cases the co-localization was not complete (Fig. 6, Fig. S8). Then, we tested whether the NLR-side BASS in the [b] prion state could induce prion aggregation of the corresponding BELL-side BASS. Strains expressing RFP-fused BELL-side motifs in the non-prion [ $b^{*}$ ] state were confronted with strains expressing the corresponding GFP-fused NLR-side motifs in the [b] prion state. In all tested cases, we found that the NLR-side BASS efficiently converts the BELL-side BASS to the prion state (Fig. 5B). The level of amino acid identity within cross-seeding BASS motif pairs is in the range of $43-56 \%$ (Fig. 2, Fig. S7) and comparable to the level of identity leading to prion crossseeding of fungal amyloid signaling motifs $[7,16,53]$. When expressed in this fungal model, tested BASS are functionally analogous to fungal amyloid signal motifs in the sense that the NLR-side motif is able to interact with the BELL-side motif and to induce its prion aggregation.

\section{BASS3 motifs cross-seed with RHIM and PP-motif in a fungal model}

The BASS3 motifs shows homology to the fungal PP and animal RHIM motifs. In particular all three motifs show a common G- $\phi-Q-\phi-G$ core region (Fig.1, Fig. S7). To analyze the functional importance of this region for BASS3 prion-formation, we have generated four proline mutants of the S. atratus BELL-side BASS3 motif on conserved position of the motif. We mutated three residues in the G- $\phi-Q-\phi-G$ core region common to BASS3, PP and RHIM (Q113P, V114P and G115P) and a conserved $Q$ residue conserved specifically in the BASS3 motif (Q120P) (Fig. S7). Wild-type and mutant BASS3 motifs fused to GFP were expressed in the $P$. anserina model and we found that all four mutations totally abolished formation of the [b] prion-state in vivo as assayed by the formation and propagation of GFP-dots (Table 5). These results indicate that the conserved position of the BASS3 motif are involved in prion formation and support the hypothesis that the BASS3, PP and RHIM-motifs are related. 
It was reported that partial in vivo prion cross-seeding occurs between fungal PP motifs and mammalian RIP1K and RIP3K RHIM sequences [26]. The level of sequence identity between fungal PP and mammalian RHIM-motifs is in the range of $20-30 \%$, comparable to the identity level between BASS3 and RHIM or PP motifs (Fig. S7). This prompted us to assay prion cross-seeding between BASS3, RHIM and PP-motifs. We analyzed the ability of RHIM or PP prions to convert BASS3 motifs to the [b] prion state and conversely the ability of BASS3 prions to convert RHIM and PP to their respective [Rhim] and $[\pi]$ prion state. To that end, strains expression BELL-side BASS3 from S. atratus or N. fusca and displaying the non-prion [ $\left.\mathrm{b}^{*}\right]$ phenotype were confronted to strain expressing mammalian [Rhim] prions (human RIP1(524-551) or human RIP3(444-496)) or fungal $[\pi]$ prions (Podospora anserina HELLP(214-271) or Chaetomium globosum HELLP(215-278)) and their prion status was analyzed following this confrontation. In the reverse experiment, the strains expressing BASS3 were used as prion-donor and the RHIM and PP-expressing strains as recipients. We find that as reported previously in the case of PP and RHIM motifs [26], there is a partial prion cross-seeding between [b] and mammalian [Rhim] and fungal $[\pi]$ prions (Fig. 7). While the spontaneous prion-conversion rate was in the range of 10 to $20 \%$ in these experiments, about 50 to $60 \%$ of the BASS3 expressing strains were converted to the $[\mathrm{b}]$ prion strain after contact with strains containing [Rhim] or $[\pi]$ prions. The rate of homotypic conversion is higher, typically in the range of 80 to $90 \%$. Both S. atratus and N. fusca BASS3 [b] prions induced [Rhim] and $[\pi$ ] prions at a similar rate of 50 to $60 \%$. BASS1 motifs were not converted to the prion state at a higher rate after contact with [Rhim] or $[\pi]$ prions indicating that cross-seeding was limited to the BASS3 motifs. These results are consistent with the side directed mutagenesis experiments indicating the functional importance of the $G-\phi-Q-\phi-G$ core region and underline a functional similarity between RHIM, PP and BASS3-motifs. We have also analyzed in vivo co-localization of BASS3 motifs with either RHIM or PP-motifs tagged with RFP and GFP. As described in the case of interaction of RHIM with fungal PP-motifs, there is a partial co-localization of BASS3 and RHIM aggregates, and BASS3 and PP-motifs aggregates (Fig. S8). It is possible to observed strains coexpressing BASS3 and RHIM in which the BASS3 is aggregated and the RHIM motif remains in the [Rhim*] state, consistent with the intermediate level of prion cross-seeding measured in crossinfection experiments (Fig. 7). The same is true in the case of BASS3 and PP co-expression but not when two BASS3 motif form different bacterial species are co-expressed again in agreement with the crossinfection experiments. In a control experiment, we found no co-localization with the unrelated BASS1 motif with either BASS3, RHIM or PP and independent formation of [Rhim], [ $\pi$ ] and [b] prions (Fig. S9).

\section{Discussion}

Amyloids have initially been identified in the context of human protein-deposition diseases and correspond to protein aggregates with a cross- $\beta$ structure [57]. The nucleated-polymerization process governing their assembly allows some amyloids to propagate their conformational state as prions [ $[58,59]$. The amyloid fold can play a variety of functional roles [60, 61]. In particular, functional amyloids have been found to be involved in signal transduction cascades controlling programmed cell death processes. In mammals, the RHIM motif direct assembly of the RIPK1/RIPK3 complex in the necroptosis pathway $[21,62]$. In fungi, amyloid motifs control a signal transduction mechanism based on transmission of an amyloid fold from an NLR protein to downstream cell-death execution proteins 
$[7,16]$. In the prokaryotic reign, functional amyloids have been found to be involved in biofilm formation, development and virulence [63-65]. We show here that phylogenetically diverse bacterial lineages including Actinobacteria and Cyanobacteria display fungal-like amyloid signaling motifs and thus suggest that NLR-associated amyloid signaling is also present in these multicellular bacterial lineages.

\section{BASS form prions}

When expressed in Podospora anserina, selected BASS motifs behaved as prion-forming domains and thus were functionally analogous to previously characterized fungal prion signaling motifs. In other words, they are capable, in vivo, in this heterologous setting, to form aggregates and to propagate this aggregation state as prions. Importantly, we find that the NLR-side and BELL-side of matching motifs are able to co-aggregate and that, NLR-side motifs convert BELL-side motifs to the prion state. These results are consistent with the proposition that the motifs functionally interact and that the NLR-side motif serves as a template for the transconformation of the BELL-side motif as shown for the fungal prion signaling motifs $[7,16,19,26]$. We find that the same motifs form fibrils in vitro. In case of the BASS1 motif of Streptomyces coelicolor, solid-state NMR analyses indicate the formation of a highly-ordered cross- $\beta$ structure. Comparable observations have been made using the same biophysical techniques for analogous fungal prion-forming domains such as HET-s(218-289) [42, 52, 66], HELLF [19], NWD2(1-30) [7] and PP [16]. For BASS motifs that were tested experimentally, we confirm the identified sequences form amyloid prions, again making is plausible that the other motifs also represent analogous prion amyloids.

\section{Relation of BASS3 to RHIM and PP}

Several families of amyloid signaling motifs were described in fungi and remarkably, PP, one such family appears to be related to the animal RHIM amyloid motifs $[16,21,26]$. We now find that a motif similar to RHIM/PP and occurring in the same domain architectures as the fungal PP-motif also exists in Bacteria. There is partial prion cross-seeding between BASS3, RHIM and PP-motifs as tested in the P. anserina model (this study and [26]). RHIM, PP and BASS3 motifs, all share the central G- $\phi-Q-$ $\phi-G$ signature (Fig. S7). This signature corresponds to the central core of the RHIM amyloid structure with the tight interdigitation of two such motifs in one $\beta$-strand layer [62]. Mutation in this core region abolish BASS3 prion formation in vivo. The consensus sequences for the mammalian, fungal and bacterial motif however differ in other positions (Fig. S7), which might explain why inter-kingdom cross-seeding is only partial and not as efficient as in homotypic interactions or intra-kingdom interactions. These results suggest some level of structural similarity between the amyloid structures of these motifs in the different kingdoms but also some major differences that should account for these intermediate levels of prion cross-seeding. The extension of the presence of this amyloid motif to prokaryotic lineages supports the hypothesis of long-term conservation of this motif for amyloid signaling purposes from bacteria, to metazoan and fungi but due to the moderate sequence similarity between the motifs the possibility of a convergent evolution towards the amyloid signaling function cannot be ruled out completely. 


\section{Diversity of amyloid signaling motifs in Bacteria}

In addition to BASS3, we have also identified nine other bacterial amyloid motif families. This finding suggests an extensive diversification of this type of signaling domains in prokaryotes. In fungi, amyloid signaling motifs were also found to be diverse with so far three main families described (HRAM, PP and sigma) $[13,15]$. Diversity of such motifs in bacterial lineages appears even greater than in fungi, which might be expected considering the larger phylogenetic breadth of the bacterial lineages compared to fungi [67]. Diversity of bacterial motifs almost certainly exceed the 10 families described herein, considering we restricted the analysis to motifs recovering at least 5 non-redundant matching gene pairs. Except for the RHIM/PP-motifs, there is no obvious sequence homology between bacterial and fungal motifs, which is not surprising considering that some of the BASS motifs appear specific for a given bacterial phylum. In spite of the lack of direct sequence homology, bacterial and fungal motifs have common features, they show a similar length (typical 20-25 amino acids) and amino acid composition biases, apparently typical of proteins forming $\beta$-arch structures [39]. In addition, some bacterial motifs appear as double (or triple) pseudo-repeats as described in the case of fungal HRAMs $[15,42]$.

\section{NLRs, BELL domains and amyloid signaling in multicellular bacteria}

Proteins with NLR domain architectures control various biotic interactions in plants, animals and fungi $[1,68]$. Throughout, we have used the designation NLR to specify proteins displaying a domain architecture associating a NB-ARC or NACHT-type NOD domain (nucleotide binding and oligomerization domain) and ANK, TPR, WD or LRR super-structure forming repeats (SSFR). While some authors reserve the NLR designation to plant and animal NBS-LRR proteins, we adhere to an extended assertion of the term also including NB-SSFR proteins found in fungi and prokaryotes. It has been reported previously that bacterial genomes (in particular in Actinobacteria and Cyanobacteria) display genes encoding proteins with a NBS-SSFR architecture [4, 36, 37]. In fungi, both NACHT/WD and NB$A R C / T P R$ proteins have been shown to control non-self recognition and programmed cell death in different species [5, 6, 8-10]. Urbach and Ausubel have shown that these NACHT/WD and NB-ARC/TPR architectures are ancestral to the NBS/LRR architecture, which represent a more recent acquisition (that occurred independently in plant and animal lineages).

In our survey of over 100000 prokaryotic genomes, we find again that NLR architecture proteins are characteristic of bacterial lineages encompassing genera and species with a multicellular organization (filamentous or multicellular aggregates forming species). These are found in filamentous Actinobacteria, Cyanobacteria and Chloroflexi and also in aggregate-forming Archaea. Characteristic of those bacterial lineages that display NLRs is also the presence of the BELL domain. Like fungal NLRs, prokaryotic NLRs typically have NB-ARC/TPR or NACHT/WD architectures and do not display LRR repeats $[3,4,69]$. Another communality between fungal and bacterial NLRs is the presence in both lineages of NLR with WD and TPR-repeats showing high levels of internal conservation (with repeat units showing 80-90\% sequence identity, a situation in stark contrast with the bulk of the WD and TPRrepeats in proteins), [3], [70, 71]. In fungi, this internal conservation is related to a mechanism of rapid diversification of repeat domain binding specificity [72]. These NLR-repeats are under positive Darwinian selection and diversify rapidly by a mechanism of repeat-unit reshuffling representing both the cause and consequence of their high internal conservation $[3,72,73]$. Analogous WD and TPR 
repeats in bacterial NLRs might similarly be under a specific evolutionary regimen allowing rapid diversification. The possibility of an ancestral role of NLR-like proteins in programmed cell death and host defense in multicellular bacteria has been raised before [37]. Together with the communalities mentioned above, the mechanistic similarity between fungal NLR/amyloid motif/HeLo and bacterial NLR/amyloid motif/BELL domain associations we report, now makes it indeed plausible to envision that the bacterial gene pairs equally function in host defense and programmed cell death. In this hypothesis, the BELL domain might correspond to a programmed cell-death execution domain. In support of this view is the sequence similarity between the signature consensus sequences in the $\mathrm{N}$ terminal $\alpha$-helical region of BELL, fungal HeLo, metazoan MLKL and plant CC-domains (Fig. 1). Functional studies of the BELL-domain and its possible function in cell death execution are now required to explore this hypothesis. Of note is also the fact that other protein domains related to immune functions and programmed cell death in animals and plants (such as the TIR and caspase-like CHAT domain) can also be found associated to amyloid signaling motifs and that the same domains are frequently found as N-terminal domain of NLRs in multicellular bacteria (Table S1).

Remarkably, in fungi NLRs are restricted to filamentous genera (both in Ascomycota and Basidiomycota) and are not found in yeast species [3]. This situation is mirrored in the present study by the fact that NLRs in general as well as the matching NLR/BELL gene pairs are found in Actinobacteria and Cyanobacteria species with a multicellular morphology but appear to be absent or rare in phylogenetically related unicellular classes. Although, PCD pathways also exist in unicellular species those continue to some extend to represent a paradox $[74,75]$. Defense-related PCD is generally considered an attribute of multicellulars in which altruistic cell death can be directly advantageous for the survival of the organism [76]. Our results suggest that filamentous fungi and bacteria have in common the use of NLR-associated amyloid signaling processes. We propose that this shared molecular mechanistic feature between filamentous fungi and filamentous Actino and Cyanobacteria stems from their common cellular organization and life style. These fungi and bacteria are phylogenetically distant but morphologically alike. In a similar way, abundance and complexity of secondary metabolism clusters involved in allelopathic interactions appears to be a specific feature of filamentous fungi and bacteria compared to related unicellular genera [35, 77, 78]. Presence of NLRs and amyloid signaling might represent a common genome hallmark of diverse multicellular microbes. As multicellular organisms, microbes from both reigns could have to cope with parasites and pathogens and thus might rely on altruistic cell suicide for defense, as an immune-related programmed cell death mechanism akin to those operating in multicellular plant and animals lineages. We propose based on these genomic and functional analogies that filamentous fungi and filamentous bacteria share in common the use of NLR-associated amyloid motifs in the control of immune-related programmed cell death. The implication of this hypothesis is that the use of NLRs for immune-related functions might be ancestral and shared universally between multicellular Archaea, Bacteria, fungi, plants and animals. The mechanistic resemblance we report between prokaryotic and eukaryotic NLRs, invites to reconsider the evolutionary trajectory of this protein family, which might have a very ancient history in the control of biotic interactions. The proposed NLR-related systems described here appear to be just one specific example among a variety of recently described NTP-dependent biological conflict systems preponderantly found in multicellular prokaryotes [79].

\section{Material and Methods}


Homology searches. The N-terminal 102 amino acid-long fragment of protein ONI86675.1 (A0A1V2QF20_9PSEU) [80] was used as the BELL domain query in Jackhmmer [81, 82] (20 iterations, 2133 hits) and Psi-blast [83, 84], (5 iterations, 502 hits) homology searches in UniProtKB [85] and NCBI $\mathrm{nr}$ databases [86](respectively), as of March 2019. In order to extend the coverage, the searches were re-run with the lowest scoring above-the-threshold Jackhmmer hits in Archaea (OPX82726.1 / A0A1V4VC50_9EURY),[87], Chloroflexi (ACL23627.1 / B8G4Z4_CHLAD) and Proteobacteria (MBN58330.1 / A0A2E7JCT6_9GAMM) [88], resulting in 2383, 2553 and 939 hits, respectively. In all cases, standard website parameters were used. Combined results consisted of 2797 non-redundant sequences including 2354 sequences shorter than 200 amino acids. The length threshold was chosen to filter out BELL homologues forming "all-in-one" NLR architectures.

Neighboring NLRs identification. All sequence identifiers in the set were mapped to NCBI accessions using UniProt mapping tool. Accessions of identical sequences in the NCBI nr database were added using the Blast database command tool [89]. This resulted in set of 2810 protein accessions from genome-wide studies, restricted to GenBank and RefSeq NP and YP series accessions. An in-house Python (version 3.5) script (aided by requests (Reitz, K., n.d.) and xmltodict (Blech, n.d.) packages) was used to query NCBI Entrez E-utils in order to fetch almost 23k proteins coded by genes within the +/5000 bp neighborhood of the genes encoding these BELL homologues. Among the proteins coded by the BELL-neighboring genes, 730 were matched with Pfam , NACHT (PF05729) [69] or NB-ARC (PF00931) [90] profiles. This included 467 sequences (426 unique) where the match bordered a relatively short $\mathrm{N}$-terminus of 10 to 150 amino acids in length (396 unique extensions), which is a typical feature of fungal NLR proteins containing amyloid signaling motifs.

Motif extraction. The C-terminal boundaries of the BELL domains were delimited according to the final Profile HMM (pHMM) of the original Jackhmmer search using the hmmalign tool of the standalone HMMER distribution (version 3.2.1) [81]. The C-terminal extensions longer than 10 amino acids were extracted. The redundancy of the set was reduced to 90\% using cdhit [91, 92], (version 4.6, standard parameters) resulting in 1814 unique sequences. The common motifs in the C-termini were extracted using MEME $[93,94]$ (version 5.0.2, standalone), allowing for motifs of any length from 6 to 30 and possibly repeated in sequence (--anr option), while requiring that each motif was found in at least 10 instances. The search yielded 66 motifs (prefixed later "all") above the standard E-value threshold of 0.1. Aiming at motifs restricted to taxonomic branches, additional MEME searches (requiring at least 5 motif instances only) were performed for taxonomic subsets of UniProtKB sequences including: Cyanobacteria (found 27 motifs in 359 sequences, prefixed later "cya"), combined Proteobacteria and Chloroflexi (5 in 69, "pch"), and Streptomycetes (19 in 608, "str"). Finally, the MEME search with the same parameters was performed in N-termini of the NLR-containing genomic neighbors of BELL domains (39 motifs found in 338 sequences, "nIr").

Motif profile generation. For each motif, a pHMM was trained using hmmbuild from the HMMER package on instances reported by MEME. Then, the pHMMs were used to re-search the BELL Cterminal domains and NLR N-terminal domains, respectively, using hmmsearch with sequence and domain E-values set to 0.01 and heuristic filters turned off for sensitivity (--max option). The resulting hits were extended each side by 5 amino acids, and then re-aligned to the pHMMs and trimmed of unaligned residues using hmmalign (--trim option). Eventually, the resulting alignments were used to train final pHMMs for the motifs again using hmmbuild with standard options. The entire procedure 
aimed at generalizing the motifs, especially gapped, which could be truncated or over-specialized by MEME.

Motif pairs identification. Motif pairs were identified whenever hits from the same profile HMM were found in BELL C-termini and their corresponding NLR(s) N-termini, using hmmsearch with sequence and domain E-value set to 0.01 and maximum sensitivity (--max option). There were 1157 such pairs (904 using BELL-side motifs and 253 using NLR-side motifs) in 315 (293 and 246, respectively) sequence pairs (there was a considerable overlap between motifs, see below). Only motifs with at least 5 unique pair hits (in terms of sequence) were considered. The criterion was met by 29 BELL-side motifs and 12 NLR-side motifs with 1087 hits in 295 sequence pairs.

Since the sequences selected for the BASS3 motif were not included neither in the GenBank nor RefSeq NP or YP series at the time of the genome mining, they were not processed for identifying neighboring NLRs, and hence are not covered in the motif pair list in Table S2. Nevertheless, the BASS3 motifs can be found in these sequences with the motif pHMMs at default identification threshold (E-value of 0.01 ), except for ACC79697.1 being slightly below (E-value of 0.1 ).

Motif clustering. Motifs were grouped based on overlapping matches (hits in the same sequence pairs). Motifs were joined if at least half of pairs hit by one motif profile HMM were also matched by the other. The procedure yielded ten motif classes termed BASS1 to 10 . For each class, the member motif matching most sequences was used as the class representative.

Motif characterization. For each member motif in classes BASS1-10, the sequence regions matched with its pHMM were extended each side by 5 amino acids and pairwisely locally aligned using the Waterman-Eggert method [95, 96] implemented in EMBOSS (matcher tool, version 6.6.0.0) [97]. The standard parameters were used including the BLOSUM62 matrix and gap opening/extending penalties 14/4. For each motif class representative, the sequence fragments from the resulting pairwise alignments were combined into multiple sequence alignments using Clustal Omega [98, 99](version 1.2.4, standalone) with the --dealign and --auto switches. The resulting MSAs were trimmed manually and profiles were generated using weblogo3 [100] (Weblogo software repository webpage, 2019).

In addition, for each motif class and sequence, the pairwise alignments were used to obtain the combined longest fragment matching any member motif. The dealigned fragments were then combined into MSA using Clustal Omega with --full and --full-iter options, curated when necessary (BASS4) and trimmed manually. Amino acid composition of such generated MSAs was then calculated using the quantiprot package [101], after removing redundant sequences. The per-class and overall (unweighted) composition was then compared with amino acid composition of non-redundant sequence sets of fungal functional amyloid motifs including Het-s Related Amyloid Motif (HRAM) [15], Pfam NACHT_sigma (PF17106) and Ses_B (PF17046, aka PP-motif) $[3,15]$, beta-solenoid repeat regions extracted from the Protein Data Bank according to RepeatsDB (only reviewed entries, as of May 2019), experimentally verified amyloid fragments from AmyLoad (as of March 2017), and intrinsically disordered protein regions from DisProt [102] (as of July 2019, only unambiguous entries). Current SwissProt amino acid statistics were used as a reference.

Highly internally conserved pairs search. Local pairwise alignments of C-termini of BELL domains and their neighboring NLR(s) N-termini were performed using the matcher tool with the standard 
parameters. For each pair ten best alternatives were filtered for minimum alignment length of 15 amino acid and minimum score of 40 . The procedure yielded in 292 hits (including 234 unique) in 283 sequence pairs. The number included 44 sequence pairs not matched with the BASS1-10 motifs, comprising 25 sequence pairs not matched with pHMM of any motif.

Phylogenetic distribution. Protein accession lists of genome assemblies listed in the Genome Taxonomy Database (GTDB) [34] metadata sheets for Bacteria (113,324 items) and Archaea (1183) were downloaded from NCBI ftp (ftp.ncbi.nlm.nih.gov/genomes/all/; as of April 2019). The accesions were matched with the ABC_tran (PF00005) [103], NACHT, NB-ARC, Beta_propeller (CL0186) [104], TPR (CL0020) [105], CHAT (PF12770) [106], PNP_UDP_1 (PF01048) [107] and TIR (PF01582) [108]Pfam profiles hit lists downloaded from Pfam ftp (ftp.ebi.ac.uk/pub/databases/Pfam/current_release/; as of February 2019). In addition, distributions of previously found BELL domain homologues and motif pairs involving their C-termini in GTDB-listed genomes were recorded. For each, the total number of hits and the number of genomes with hits is provided. The sequence accession redundancy was resolved with the NCBI Blast nr database (as of March 2019).

Strains and plasmids. To avoid interference with endogenous prions, the $P$. anserina $\Delta$ het-s

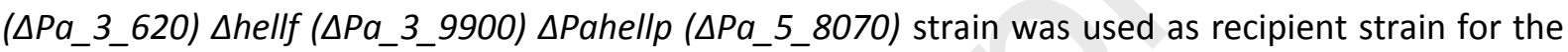
expression of molecular fusions of BASS motifs and the GFP (green fluorescent protein) or RFP (red fluorescent protein). These fusions were expressed either from a plasmid based on the pGEM-T backbone (Promega) named pOP plasmid [16], containing RFP, or from a derivative of the pAN52.1 GFP vector [109], named pGB6-GFP plasmid containing GFP. In both cases the molecular fusions were under the control of the constitutive $P$. anserina gpd (glyceraldehyde-3-phosphate dehydrogenase) promoter. The $\Delta$ Pahellp $\Delta$ het-s $\Delta$ hellf strain was transformed as described [110] with one or two fusion constructs along with a vector carrying a phleomycin-resistance gene ble, pPaBle (using a 10:1 molar ratio). Phleomycin-resistant transformants were selected, grown for $30 \mathrm{~h}$ at $26^{\circ} \mathrm{C}$ and screened for the expression of the transgenes using a fluorescence microscope. Fragments (protein position indicated in brackets) of the following genes (accessions from GenBank or RefSeq) were amplified using specific primers: CAB66307.1 (110-139), CAB66306.1 (1-34), WP_037701008.1 (70-124), WP_037701012.1 (137), ACC79696.1 (94-126), ACC79697.1 (1-38), WP_063130184.1 (74-128). The PCR products were cloned upstream of the RFP coding sequence in the pOP plasmid using $\mathrm{Pacl} / \mathrm{Bg} / \mathrm{ll}$ restriction enzymes or downstream of the GFP in the pAN52.1 plasmid using Notl/BamHI restriction enzymes. For heterologous expression in $E$. coli, the following fragments were amplified using specific primers and cloned in pET24a (Novagen) using the Ndel/Xhol restrictions sites: CAB66307.1 (110-139) or (38-139), WP_037701008.1 (97-124), ACC79696.1 (94-126), WP_063130184.1 (100-128). RIP1 and RIP3 RHIM motif fusions as well as fungal PP-motif fusion proteins used in prion cross-seeding experiments are described in [26]. Site directed mutagenesis was performed on the pAN52 GFP vector containing WP_037701008.1 (70-124) (B3-B S.a.). We obtained 4 plasmids expressing products bearing the following specific single amino acid mutations: Q113P, V114P, G115P or Q120P. These modified vectors were used to transform the $P$. anserina $\Delta$ het-s $\Delta$ hellf $\Delta$ hellp strain and transformants were monitored in fluorescence microscopy for the presence of dots, 4, 8 and 15 days after transfection or four days after contact with a strain expressing wild-type GFP-B3-B S.a. in [b] prion state.

Prion propagation. The [b] phenotype (acquisition of the [b] prion) was defined in Podospora strains expressing fluorescent fusion proteins as the absence $\left(\left[\mathrm{b}^{*}\right]\right)$ or the presence ([b]) of fluorescent dotlike aggregates. To monitor the propagation of the [b] prion, prion free strains were subcultured in 
presence of [b] prion donor strain and after $72 \mathrm{~h}$ (contact between tested and donor strains was established after $24 \mathrm{~h}$ of subculture) the initially prion free tested strain was subcultured on fresh medium and monitored for the presence of aggregates using fluorescence microscopy.

Protein preparation and fibril formation. 6his-tagged proteins were expressed from pET24a constructs in E. coli BL21-CodonPlus ${ }^{\circledR}$-RP competent cells as insoluble proteins and purified under denaturing conditions using its terminal 6 histidine tag as previously described [111]. Briefly, cells were grown at $37^{\circ} \mathrm{C}$ in DYT medium to $0.6 \mathrm{OD}_{600}$ and expression was induced with $1 \mathrm{mM}$ isopropyl $\beta$-D-1thiogalactopyranoside. After, $4 \mathrm{~h}$, cells were harvested by centrifugation, frozen at $-80^{\circ} \mathrm{C}$ sonicated on ice in a lysis buffer (Tris $50 \mathrm{mM}, 150 \mathrm{mM} \mathrm{NaCl}, \mathrm{pH}$ 8) and centrifuged for $20 \mathrm{~min}$ at 20,000 g to remove E. coli contaminants. The pellet was washed in the same buffer and resuspended in denaturing buffer (6M guanidinium $\mathrm{HCl}, 150 \mathrm{mM} \mathrm{NaCl}$, and $100 \mathrm{mM}$ Tris- $\mathrm{HCl}, \mathrm{pH}$ 8) until complete solubilization. The lysate was incubated with Talon Resin (CLONTECH) for $1 \mathrm{~h}$ at $20^{\circ} \mathrm{C}$, and the resin was extensively washed with $8 \mathrm{M}$ urea, $150 \mathrm{mM} \mathrm{NaCl}$, and $100 \mathrm{mM}$ Tris $-\mathrm{HCl}, \mathrm{pH}$ 8. The protein were eluted from the resin in the same buffer containing $200 \mathrm{mM}$ imidazole. The proteins were pure as judged by sodiumdodecyl-sulfate polyacrylamide-gel electrophoreses (SDS-PAGE) followed by Coomassie-Blue staining and yield was in the range of $\sim 2-4 \mathrm{mg}$ of protein per liter of culture. To eliminate urea, elution buffer was replaced by overnight dialysis at $4^{\circ} \mathrm{C}$ against Milli-Q water. Fibrills formation resulted spontaneously from dialysis process followed by sample storage in $\mathrm{H}_{2} \mathrm{O}$ or in ammonium acetate buffer $100 \mathrm{mM} \mathrm{pH} 4.5$ at $4^{\circ} \mathrm{C}$ for 7 to 30 days.

Light Microscopy. $P$. anserina hyphae were inoculated on solid medium and cultivated for 24 to $48 \mathrm{~h}$ at $26^{\circ} \mathrm{C}$. The medium was then cut out, placed on a glass slide and examined with a Leica DMRXA microscope equipped with a Micromax CCD (Princeton Instruments) controlled by the Metamorph 5.06 software (Roper Scientific). The microscope was fitted with a Leica PL APO 100X immersion lens.

Transmission Electron Microscopy. For fibrils observations, negative staining was performed: aggregated proteins were adsorbed onto Formvar-coated copper grids (400 mesh) and allowed to dry for $15 \mathrm{~min}$ in air, grids were then negatively stained $1 \mathrm{~min}$ with $10 \mu \mathrm{L}$ of freshly prepared $2 \%$ uranyl acetate in water, dried with filter paper, and examined with a Hitachi $\mathrm{H7650}$ transmission electron microscope (Hitachi, Krefeld, Germany) at an accelerating voltage of $120 \mathrm{kV}$. TEM was performed at the Pôle Imagerie Électronique of the Bordeaux Imaging Center using a Gatan USC1000 2k x 2k camera.

Solid-state NMR of BASS1 fibrils. The solid-state NMR spectrum of BASS 1 was recorded at $800 \mathrm{MHz}$ on a Bruker Biospin magnet using a triple resonance $3.2 \mathrm{~mm}$ probe at a spinning frequency of $11 \mathrm{kHz}$. 64 scans were used using a recycle delay of $3 \mathrm{sec}$ and an acquisition time of $17 \mathrm{~ms}$.

X-ray diffraction of BASS1 and BASS3 fibrils. Fiber diffraction pattern was measured at $4^{\circ} \mathrm{C}$ on an Excillum MetalJet $X$-ray generator at the galium wavelength $(K \alpha, \lambda=1.34 \AA$ ). The source was equipped with Xenocs Fox3D optics and a Dectris Eiger $1 \mathrm{M}$ detector on a $2 \theta$ arm of a STOE stadivari goniometer. The detector has been turned by $90^{\circ}$ to have the blind region vertical to hide as much as possible the shadow of the beamstop. The viscous concentrated hydrated sample was mounted in a MicroLoop ${ }^{\mathrm{TM}}$ from Mitegen on a goniometer head under the cold nitrogen flow. The diffraction pattern corresponds to a $360^{\circ}$ rotation along the phi axis with an exposure time of $180 \mathrm{sec}$.

ThT fluorescence assay. The aggregated bacterial amyloid fibril samples and BSA (at $2 \mu \mathrm{g} / \mathrm{ul}$ ), supplied 
with $20 \mu \mathrm{M}$ ThT were transferred to a 96 well corning plate (bottom transparent) with $100 \mu \mathrm{L} /$ well. The fluorescence measurements were carried out on three independent samples in a CLARIOstar ${ }^{\circledR}$ Plus plate reader with ten flashes per well (excitation wavelength $440 \mathrm{~nm}$ and emission wavelength 480 $\mathrm{nm})$. Ten independent measurements are averaged and plotted per sample.

\section{Acknowledgements}

The work was funded by recurrent funds from CNRS and University of Bordeaux to SJS and AL and an ANR grant to SJS and AL (ANR SFAS AAP CE11). WD was supported in part by the National Science Centre of Poland (grant no. 2015/17/D/ST6/04054). AD was supported by the Nouvelle Aquitaine Regional Council. TB was supported by the Ministère de la Recherche et de l'Enseignement Supérieur. We thanks Jayakrishna Shenoy for assistance with the ThT-binding experiments. 


\section{Figure Captions}

Figure 1. RHIM-like motifs in bacteria are associated to BELL domains. A. Genome and domain architecture of the ONI86675.1 and ONI86674.1 gene pair from the actinobacterium strain Saccharothrix sp. ALI-22-I. The relative orientation and the overlap between the two ORF are given (the two ORF overlap by $4 \mathrm{bp}$ ) as well as the size and domain architecture of the corresponding proteins, respectively for a BELL-domain protein with a C-terminal RHIM-like motif and a NLR-related protein with a NB-ARC and TPR repeat domain and an N-terminal RHIM-like motif. The RHIM-like motif are represented in red in the ORF diagram and the protein cartoon. B. Alignment of the RHIM-like motifs of the proteins encoded by the ONI86675.1 and ONI86674.1 gene pair and the RHIM-motif of the human RIP1 and RIP3 kinases. C. Alignment of BELL-domains from various prokaryotes (Strep, Q9RDG0 from Streptomyces coelicolor A3(2) ; Chlor, HBY96210.1 from Chloroflexi bacterium ; Sacch, ONI86675.1 from Saccharothrix sp. ALI-22-I; Flavo, SDZ50707.1 from Flavobacterium aquidurense ; Nosto, RCJ33357.1 from Nostoc punctiforme NIES-2108; Granu, ROP69996.1 from Granulicella sp. GAS466 ; Metha, AEB69174.1 from Methanothrix soehngenii (strain ATCC 5969) ; Magne, ETR68090.1 from Candidatus Magnetoglobus multicellularis str. Araruama). D. Secondary structure prediction for ONI86675.1 from Saccharothrix sp. ALI-22-I, red bars represent $\alpha$-helical propensity, blue bar $\beta$-sheet propensity. Boxing corresponds to the $\alpha$-helices predicted in the contact-based model given in F. Note that secondary structure prediction merged modelled helices 1 and 2 of the contact-based model. E. Consensus sequence of the $25 \mathrm{~N}$-terminal residues of the BELL-domain and other predicted or known cell death execution domains in fungi, plants and mammals. The consensus sequence was generated with Weblogo from a HHMER alignment using the following sequences as queries : ONI86675.1 (BELL), C. globosum HELLP (Hell), P. anserina, HET-S (HeLo), mouse MLKL (MLKL) and Arabidopsis thaliana RPW8.1 (RPW8). F. Homology model of the BELL-domain of ONI86675.1 from Saccharothrix sp. ALI22- based on a contact map (model generated by RAPTOR-X contact).

Figure 2. Ten bacterial amyloid signaling sequences motifs (BASS). For each of the ten identified motifs, a representative gene pair is given. A consensus sequence for the motif is given. The consensus was generated using Weblogo from the alignment of all motifs pairs bearing the corresponding motif. For each gene pair chosen as illustrative example, the species name, the genome architecture, the gene number and protein size are given as well as an alignment of the BELL-domain and NLR-associate motifs. The number given above the gene diagram is the distance between the BELL-domain encoding and NLR encoding ORF, negative number represent gene overlaps. The sequences encoding the BASS motif is represented in red, the BELL-domain in grey, NB-ARC domain is light blue, TPR repeats in dark blue and WD repeats in green. Under the alignment the secondary structure prediction for the individual sequences of the BELL-domain motif (Jpred) or for a HMM-alignment of the sequence (Jpred $(H M M))$ are given ( $E$ for extended, $H$ for helical). The number of motif pairs identified with the different motifs is given in parentheses. The dots symbolize the phylogenetic distribution of the motif (green, cyanobacteria, beige, actinobacteria).

Figure 3. BASS-motifs form fibrils in vitro. A. Electron micrographs of fibrils formed by selected BASS1 and BASS3 motifs as indicated (scale bar, 100nm). B. ThT-fluorescence signal of BASS1 and BASS3 fibrils. BASS1 or BASS3 fibrils (Streptomyces coelicolor A3(2) BASS1 motif from CAB66307.1, residue 38 to 139, and Nocardia fusca BASS3 motif from WP_063130184.1, residue 74-128), a no protein control 
(NC) and BSA were incubated with ThT and fluorescence was measured (excitation wavelength $440 \mathrm{~nm}$ and emission wavelength $480 \mathrm{~nm}$ ). C. X-ray diffraction pattern of unoriented BASS1 fibrils (Streptomyces coelicolor A3(2) BASS1 motif from CAB66307.1, residue 38 to 139) are given, the reflection at $4.7 \AA$ is marked by an arrow. D. ${ }^{13} \mathrm{C}$ solid-state NMR spectrum of BASS 1 fibrils (Streptomyces coelicolor A3(2) BASS1 motif from CAB66307.1, residue 38 to 139).

Figure 4. Expression of GFP and RFP-fused BASS-motifs in Podospora anserina. Micrographs of $P$. anserina strains expressing molecular fusions of BASS1 and BASS3 motifs with GFP (in N-terminus) or RFP (in C-terminus) as indicated above each micrograph (Scale bar $5 \mu \mathrm{m}$ ). Transformants displayed an initial diffuse fluorescence noted $\left[\mathrm{b}^{*}\right]$ phenotype (left side of the panels) and acquired dot-like fluorescent aggregates, [b] phenotype, after contact with strains already spontaneously expressing the infectious aggregated [b] prion state (right side of the panels).

Figure 5. BASS motifs propagate as prions in Podospora anserina and NLR-side motifs induce prion formation of BELL-side motifs. A. Histogram representing the percentage of [ $\left.\mathrm{b}^{*}\right]$ strains expressing the given BASS motifs (fused to RFP in C-terminus) converted to [b] prion phenotype after contact, either with a non-transfected prion-free control strain (spontaneous, in blue), or with [b] prion strains expressing the same motif in the aggregated state (induced, in orange). Percentages are expressed as the mean value \pm standard deviation for three independent experiments using six subcultures of four different transformants and corresponding to the analysis of $\sim 70$ subcultures for each BASS motif. In each case, the BASS motif are fused to RFP. B. Histogram representing the percentage of [ $\left.b^{*}\right]$ strains expressing the given BASS motif (fused to RFP in C-terminus) as indicated, converted to the [b] prion phenotype by contact either with non-transfected prion-free control strains (spontaneous, in blue), or with strains expressing the corresponding NLR-side motif fused to GFP (in N-terminus) in the aggregated [b] prion state (induced, in orange). Percentages are expressed as the mean value \pm standard deviation for experiments using six to twelve subcultures of six to ten different transformants and correspond to the analysis of 80 to 100 subcultures for each BASS motif.

Figure 6. BELL and NLR-side BASS motif co-localize in Podospora anserina. Micrographs of Podospora anserina strains co-expressing BELL-side BASS motifs fused to GFP (in N-terminus) as indicated and the corresponding NLR-side motif fused to RFP (in C-terminus), (Scale bar $5 \mu \mathrm{m}$ ). Panel are from left to right, bright field, RFP, GFP and overlay.

Figure 7. The BASS3 motif shows partial prion cross-induction with fungal PP and mammalian RHIMmotifs in Podospora anserina. Histogram representing the percentage of $[b]$, [p] and [Rhim] prion containing strains obtained after contact of induced strains (initially displaying [ $\left.\mathrm{b}^{*}\right],\left[\mathrm{p}^{*}\right]$ or $[\mathrm{Rhim} *$ ] phenotype) with a $\Delta$ hellp $\Delta$ het-s $\Delta$ hellf prion-free control strain (non transfected), or prion containing strains as indicated. Phenotype after induction were determined by monitoring the acquisition of dotlike aggregates by fluorescence microscopy as an indicator of the prion formation. Percentages of prion formation were expressed as the mean value \pm standard deviation for 3 independent experiments on at least 4 different transformants corresponding to the analysis of 42 to 102 subcultures per prion 
motif combination. PP to RHIM prion cross-conversion results are as in [26] and are given here for comprehensiveness. The star sign $(*)$ refer to the fact that PaHELLP(214-271) seeding by CgHELLP(215278) was not tested.

[1] Jones JD, Vance RE, Dangl JL. Intracellular innate immune surveillance devices in plants and animals. Science. 2016;354.

[2] Mermigka G, Amprazi M, Mentzelopoulou A, Amartolou A, Sarris PF. Plant and Animal Innate Immunity Complexes: Fighting Different Enemies with Similar Weapons. Trends Plant Sci. 2019.

[3] Dyrka W, Lamacchia M, Durrens P, Kobe B, Daskalov A, Paoletti M, et al. Diversity and Variability of NOD-Like Receptors in Fungi. Genome biology and evolution. 2014;6:3137-58.

[4] Urbach JM, Ausubel FM. The NBS-LRR architectures of plant R-proteins and metazoan NLRs evolved in independent events. Proc Natl Acad Sci U S A. 2017;114:1063-8.

[5] Chevanne D, Bastiaans E, Debets A, Saupe SJ, Clave C, Paoletti M. Identification of the het-r vegetative incompatibility gene of Podospora anserina as a member of the fast evolving HNWD gene family. Curr Genet. 2009;55:93-102.

[6] Choi GH, Dawe AL, Churbanov A, Smith ML, Milgroom MG, Nuss DL. Molecular characterization of vegetative incompatibility genes that restrict hypovirus transmission in the chestnut blight fungus Cryphonectria parasitica. Genetics. 2012;190:113-27.

[7] Daskalov A, Habenstein B, Martinez D, Debets AJ, Sabate R, Loquet A, et al. Signal transduction by a fungal NOD-like receptor based on propagation of a prion amyloid fold. PLoS Biol.

2015;13:e1002059.

[8] Espagne E, Balhadere P, Penin ML, Barreau C, Turcq B. HET-E and HET-D Belong to a New Subfamily of WD40 Proteins Involved in Vegetative Incompatibility Specificity in the Fungus Podospora anserina. Genetics. 2002;161:71-81.

[9] Heller J, Clave C, Gladieux P, Saupe SJ, Glass NL. NLR surveillance of essential SEC-9 SNARE proteins induces programmed cell death upon allorecognition in filamentous fungi. Proc Natl Acad Sci U S A. 2018.

[10] Saupe S, Turcq B, Begueret J. A gene responsible for vegetative incompatibility in the fungus Podospora anserina encodes a protein with a GTP-binding motif and G beta homologous domain. Gene. 1995;162:135-9.

[11] Loquet A, Saupe SJ. Diversity of Amyloid Motifs in NLR Signaling in Fungi. Biomolecules. 2017;7.

[12] Cai X, Chen J, Xu H, Liu S, Jiang QX, Halfmann R, et al. Prion-like polymerization underlies signal transduction in antiviral immune defense and inflammasome activation. Cell. 2014;156:1207-22.

[13] Daskalov A, Paoletti M, Ness F, Saupe SJ. Genomic clustering and homology between HET-S and the NWD2 STAND protein in various fungal genomes. PloS One. 2012;7:e34854.

[14] Cai X, Xu H, Chen ZJ. Prion-Like Polymerization in Immunity and Inflammation. Cold Spring Harb Perspect Biol. 2016.

[15] Daskalov A, Dyrka W, Saupe SJ. Theme and variations: evolutionary diversification of the HET-S functional amyloid motif. Scientific reports. 2015;5:12494.

[16] Daskalov A, Habenstein B, Sabate R, Berbon M, Martinez D, Chaignepain S, et al. Identification of a novel cell death-inducing domain reveals that fungal amyloid-controlled programmed cell death is related to necroptosis. Proc Natl Acad Sci U S A. 2016;113:2720-5.

[17] Wasmer C, Lange A, Van Melckebeke H, Siemer AB, Riek R, Meier BH. Amyloid fibrils of the HET$s(218-289)$ prion form a beta solenoid with a triangular hydrophobic core. Science. 2008;319:1523-6.

[18] Wasmer C, Zimmer A, Sabate R, Soragni A, Saupe SJ, Ritter C, et al. Structural similarity between the prion domain of HET-s and a homologue can explain amyloid cross-seeding in spite of limited sequence identity. J Mol Biol. 2010;402:311-25. 
[19] Daskalov A, Martinez D, Coustou V, El Mammeri N, Berbon M, Andreas LB, et al. Structural and molecular basis of cross-seeding barriers in amyloids. bioRxiv. 2020;2020.07.06.188508.

[20] Graziani S, Silar P, Daboussi MJ. Bistability and hysteresis of the 'Secteur' differentiation are controlled by a two-gene locus in Nectria haematococca. BMC Biol. 2004;2:18.

[21] Li J, McQuade T, Siemer AB, Napetschnig J, Moriwaki K, Hsiao YS, et al. The RIP1/RIP3 necrosome forms a functional amyloid signaling complex required for programmed necrosis. Cell. 2012;150:33950.

[22] Sun X, Yin J, Starovasnik MA, Fairbrother WJ, Dixit VM. Identification of a novel homotypic interaction motif required for the phosphorylation of receptor-interacting protein (RIP) by RIP3. J Biol Chem. 2002;277:9505-11.

[23] Kleino A, Ramia NF, Bozkurt G, Shen Y, Nailwal H, Huang J, et al. Peptidoglycan-Sensing Receptors Trigger the Formation of Functional Amyloids of the Adaptor Protein Imd to Initiate Drosophila NF-kappaB Signaling. Immunity. 2017;47:635-47 e6.

[24] Pham CL, Shanmugam N, Strange M, O'Carroll A, Brown JW, Sierecki E, et al. Viral M45 and necroptosis-associated proteins form heteromeric amyloid assemblies. EMBO Rep. 2019;20.

[25] Kajava AV, Klopffleisch K, Chen S, Hofmann K. Evolutionary link between metazoan RHIM motif and prion-forming domain of fungal heterokaryon incompatibility factor HET-s/HET-s. Scientific reports. 2014;4:7436.

[26] Bardin T, Daskalov A, Barrouilhet S, Granger-Farbos A, Salin B, Blancard C, et al. Prion crossseeding between fungal and mammalian amyloid signaling motifs. bioRxiv 20200722215483. 2020.

[27] Seuring C, Greenwald J, Wasmer C, Wepf R, Saupe SJ, Meier BH, et al. The mechanism of toxicity in HET-S/HET-s prion incompatibility. PLoS Biol. 2012;10:e1001451.

[28] Greenwald J, Buhtz C, Ritter C, Kwiatkowski W, Choe S, Maddelein ML, et al. The mechanism of prion inhibition by HET-S. Mol Cell. 2010;38:889-99.

[29] Murphy JM, Czabotar PE, Hildebrand JM, Lucet IS, Zhang JG, Alvarez-Diaz S, et al. The pseudokinase MLKL mediates necroptosis via a molecular switch mechanism. Immunity.

2013;39:443-53.

[30] Wang J, Hu M, Wang J, Qi J, Han Z, Wang G, et al. Reconstitution and structure of a plant NLR resistosome conferring immunity. Science. 2019;364.

[31] Rebsamen M, Heinz LX, Meylan E, Michallet MC, Schroder K, Hofmann K, et al. DAI/ZBP1 recruits RIP1 and RIP3 through RIP homotypic interaction motifs to activate NF-kappaB. EMBO Rep.

2009;10:916-22.

[32] El-Gebali S, Mistry J, Bateman A, Eddy SR, Luciani A, Potter SC, et al. The Pfam protein families database in 2019. Nucleic Acids Research. 2018;47:D427-D32.

[33] Adachi H, Contreras M, Harant A, Wu CH, Derevnina L, Sakai T, et al. An N-terminal motif in NLR immune receptors is functionally conserved across distantly related plant species. eLife. 2019;8.

[34] Parks DH, Chuvochina M, Waite DW, Rinke C, Skarshewski A, Chaumeil PA, et al. A standardized bacterial taxonomy based on genome phylogeny substantially revises the tree of life. Nat Biotechnol. 2018;36:996-1004.

[35] Shih PM, Wu D, Latifi A, Axen SD, Fewer DP, Talla E, et al. Improving the coverage of the cyanobacterial phylum using diversity-driven genome sequencing. Proc Natl Acad Sci U S A. 2013;110:1053-8.

[36] Asplund-Samuelsson J, Bergman B, Larsson J. Prokaryotic caspase homologs: phylogenetic patterns and functional characteristics reveal considerable diversity. PLoS One. 2012;7:e49888.

[37] Koonin EV, Aravind L. Origin and evolution of eukaryotic apoptosis: the bacterial connection. Cell Death Differ. 2002;9:394-404.

[38] Fukuda Y, Nakayama Y, Tomita M. On dynamics of overlapping genes in bacterial genomes. Gene. 2003;323:181-7.

[39] Baxa U, Cassese T, Kajava AV, Steven AC. Structure, function, and amyloidogenesis of fungal prions: filament polymorphism and prion variants. Adv Protein Chem. 2006;73:125-80.

[40] Ahmed AB, Kajava AV. Breaking the amyloidogenicity code: Methods to predict amyloids from amino acid sequence. Febs Letters. 2013;587:1089-95. 
[41] Ahmed AB, Znassi N, Chateau MT, Kajava AV. A structure-based approach to predict predisposition to amyloidosis. Alzheimer's \& dementia : the journal of the Alzheimer's Association. 2014.

[42] Ritter C, Maddelein ML, Siemer AB, Luhrs T, Ernst M, Meier BH, et al. Correlation of structural elements and infectivity of the HET-s prion. Nature. 2005;435:844-8.

[43] Nimma S, Ve T, Williams SJ, Kobe B. Towards the structure of the TIR-domain signalosome. Curr Opin Struct Biol. 2017;43:122-30.

[44] O'Neill LA, Bowie AG. The family of five: TIR-domain-containing adaptors in Toll-like receptor signalling. Nat Rev Immunol. 2007;7:353-64.

[45] Freihat LA, Wheeler JI, Wong A, Turek I, Manallack DT, Irving HR. IRAK3 modulates downstream innate immune signalling through its guanylate cyclase activity. Scientific reports. 2019;9:15468.

[46] Xue JY, Wang Y, Wu P, Wang Q, Yang LT, Pan XH, et al. A primary survey on bryophyte species reveals two novel classes of nucleotide-binding site (NBS) genes. PLoS One. 2012;7:e36700.

[47] Burroughs AM, Aravind L. Identification of uncharacterized components of prokaryotic immune systems and their diverse eukaryotic reformulations. J Bacteriol. 2020.

[48] Sabate R, Baxa U, Benkemoun L, Sanchez de Groot N, Coulary-Salin B, Maddelein ML, et al. Prion and non-prion amyloids of the HET-s prion forming domain. J Mol Biol. 2007;370:768-83.

[49] Sunde M, Serpell LC, Bartlam M, Fraser PE, Pepys MB, Blake CC. Common core structure of amyloid fibrils by synchrotron X-ray diffraction. J Mol Biol. 1997;273:729-39.

[50] Loquet A, El Mammeri N, Stanek J, Berbon M, Bardiaux B, Pintacuda G, et al. 3D structure determination of amyloid fibrils using solid-state NMR spectroscopy. Methods. 2018;138-139:26-38.

[51] Wang Y, Jardetzky O. Probability-based protein secondary structure identification using combined NMR chemical-shift data. Protein Sci. 2002;11:852-61.

[52] Siemer AB, Ritter C, Ernst M, Riek R, Meier BH. High-resolution solid-state NMR spectroscopy of the prion protein HET-s in its amyloid conformation. Angew Chem Int Ed Engl. 2005;44:2441-4.

[53] Benkemoun L, Ness F, Sabate R, Ceschin J, Breton A, Clave C, et al. Two structurally similar fungal prions efficiently cross-seed in vivo but form distinct polymers when coexpressed. Mol Microbiol. 2011;82:1392-405.

[54] Benkemoun L, Sabate R, Malato L, Dos Reis S, Dalstra H, Saupe SJ, et al. Methods for the in vivo and in vitro analysis of [Het-s] prion infectivity. Methods. 2006;39:61-7.

[55] Coustou-Linares V, Maddelein ML, Begueret J, Saupe SJ. In vivo aggregation of the HET-s prion protein of the fungus Podospora anserina. Mol Microbiol. 2001;42:1325-35.

[56] Balguerie A, Dos Reis S, Ritter C, Chaignepain S, Coulary-Salin B, Forge V, et al. Domain organization and structure-function relationship of the HET-s prion protein of Podospora anserina.

Embo J. 2003;22:2071-81.

[57] Riek R, Eisenberg DS. The activities of amyloids from a structural perspective. Nature.

2016;539:227-35.

[58] Colby DW, Prusiner SB. Prions. Cold Spring Harb Perspect Biol. 2011;3:a006833.

[59] Wickner RB, Edskes HK, Gorkovskiy A, Bezsonov EE, Stroobant EE. Yeast and Fungal Prions:

Amyloid-Handling Systems, Amyloid Structure, and Prion Biology. Adv Genet. 2016;93:191-236.

[60] Baudin A, Guichard A, Collie GW, Rousseau S, Chaignepain S, Hocquellet A, et al. (1)H, (13)C,

(15)N NMR resonance assignments and secondary structure determination of the extra-cellular

domain from the human proapoptotic TRAIL-R2 death receptor 5 (DR5-ECD). Biomol NMR Assign.

2018;12:309-14.

[61] Otzen D, Riek R. Functional Amyloids. Cold Spring Harb Perspect Biol. 2019.

[62] Mompean M, Li W, Li J, Laage S, Siemer AB, Bozkurt G, et al. The Structure of the Necrosome RIPK1-RIPK3 Core, a Human Hetero-Amyloid Signaling Complex. Cell. 2018;173:1244-53 e10.

[63] Erskine E, MacPhee CE, Stanley-Wall NR. Functional Amyloid and Other Protein Fibers in the Biofilm Matrix. J Mol Biol. 2018;430:3642-56.

[64] Van Gerven N, Van der Verren SE, Reiter DM, Remaut H. The Role of Functional Amyloids in Bacterial Virulence. J Mol Biol. 2018;430:3657-84. 
[65] Rouse SL, Matthews SJ, Dueholm MS. Ecology and Biogenesis of Functional Amyloids in Pseudomonas. J Mol Biol. 2018;430:3685-95.

[66] Wan W, Stubbs G. Fungal prion HET-s as a model for structural complexity and self-propagation in prions. Proc Natl Acad Sci U S A. 2014;111:5201-6.

[67] Hug LA, Baker BJ, Anantharaman K, Brown CT, Probst AJ, Castelle CJ, et al. A new view of the tree of life. Nat Microbiol. 2016;1:16048.

[68] Uehling J, Deveau A, Paoletti M. Do fungi have an innate immune response? An NLR-based comparison to plant and animal immune systems. PLoS Pathog. 2017;13:e1006578.

[69] Koonin EV, Aravind L. The NACHT family - a new group of predicted NTPases implicated in apoptosis and MHC transcription activation. Trends in Biochemical Sciences. 2000;25:223-4.

[70] Marold JD, Kavran JM, Bowman GD, Barrick D. A Naturally Occurring Repeat Protein with High Internal Sequence Identity Defines a New Class of TPR-like Proteins. Structure. 2015;23:2055-65.

[71] Hu XJ, Li T, Wang Y, Xiong Y, Wu XH, Zhang DL, et al. Prokaryotic and Highly-Repetitive WD40 Proteins: A Systematic Study. Scientific reports. 2017;7:10585.

[72] Paoletti M, Saupe SJ, Clave C. Genesis of a fungal non-self recognition repertoire. Plos One. 2007;2:e283.

[73] Chevanne D, Saupe SJ, Clave C, Paoletti M. WD-repeat instability and diversification of the Podospora anserina hnwd non-self recognition gene family. BMC Evol Biol. 2010;10:134.

[74] Durand PM, Sym S, Michod RE. Programmed Cell Death and Complexity in Microbial Systems. Curr Biol. 2016;26:R587-R93.

[75] Koonin EV, Krupovic M. Origin of programmed cell death from antiviral defense? Proc Natl Acad Sci U S A. 2019;116:16167-9.

[76] Iranzo J, Lobkovsky AE, Wolf $\mathrm{YI}$, Koonin EV. Virus-host arms race at the joint origin of multicellularity and programmed cell death. Cell Cycle. 2014;13:3083-8.

[77] van der Meij A, Worsley SF, Hutchings MI, van Wezel GP. Chemical ecology of antibiotic production by actinomycetes. FEMS Microbiol Rev. 2017;41:392-416.

[78] Keller NP. Fungal secondary metabolism: regulation, function and drug discovery. Nat Rev Microbiol. 2019;17:167-80.

[79] Kaur G, Burroughs AM, lyer LM, Aravind L. Highly regulated, diversifying NTP-dependent biological conflict systems with implications for the emergence of multicellularity. Elife. 2020;9. [80] Yeager CM, Gallegos-Graves LV, Dunbar J, Hesse CN, Daligault H, Kuske CR. Polysaccharide Degradation Capability of Actinomycetales Soil Isolates from a Semiarid Grassland of the Colorado Plateau. Applied and Environmental Microbiology. 2017;83:e03020-16.

[81] Eddy SR. Accelerated Profile HMM Searches. PLoS computational biology. 2011;7:e1002195. [82] Potter SC, Luciani A, Eddy SR, Park Y, Lopez R, Finn RD. HMMER web server: 2018 update. Nucleic Acids Research. 2018;46:W200-W4.

[83] Altschul SF, Madden TL, Schäffer AA, Zhang J, Zhang Z, Miller W, et al. Gapped BLAST and PSIBLAST: a new generation of protein database search programs. Nucleic Acids Research. 1997;25:3389-402.

[84] Madeira F, Park Ym, Lee J, Buso N, Gur T, Madhusoodanan N, et al. The EMBL-EBI search and sequence analysis tools APIs in 2019. Nucleic Acids Research. 2019;47:W636-W41.

[85] Consortium TU. UniProt: a worldwide hub of protein knowledge. Nucleic Acids Research. 2018;47:D506-D15.

[86] Coordinators NR. Database resources of the National Center for Biotechnology Information. Nucleic Acids Research. 2018;46:D8-D13.

[87] Nobu MK, Narihiro T, Liu M, Kuroda K, Mei R, Liu W-T. Thermodynamically diverse syntrophic aromatic compound catabolism. Environmental Microbiology. 2017;19:4576-86.

[88] Tully BJ, Graham ED, Heidelberg JF. The reconstruction of 2,631 draft metagenome-assembled genomes from the global oceans. Scientific Data. 2018;5:170203.

[89] Camacho C, Coulouris G, Avagyan V, Ma N, Papadopoulos J, Bealer K, et al. BLAST+: architecture and applications. BMC bioinformatics. 2009;10:421. 
[90] van der Biezen EA, Jones JD. The NB-ARC domain: a novel signalling motif shared by plant resistance gene products and regulators of cell death in animals. Current biology: CB. 1998;8:R226-7. [91] Li W, Godzik A. Cd-hit: a fast program for clustering and comparing large sets of protein or nucleotide sequences. Bioinformatics (Oxford, England). 2006;22:1658-9.

[92] Fu L, Niu B, Zhu Z, Wu S, Li W. CD-HIT: accelerated for clustering the next-generation sequencing data. Bioinformatics (Oxford, England). 2012;28:3150-2.

[93] Bailey TL, Elkan C. Fitting a mixture model by expectation maximization to discover motifs in biopolymers. Proceedings International Conference on Intelligent Systems for Molecular Biology. 1994;2:28-36.

[94] Bailey TL, Boden M, Buske FA, Frith M, Grant CE, Clementi L, et al. MEME Suite: tools for motif discovery and searching. Nucleic Acids Research. 2009;37:W202-W8.

[95] Waterman MS, Eggert M. A new algorithm for best subsequence alignments with application to tRNA-rRNA comparisons. Journal of Molecular Biology. 1987;197:723-8.

[96] Huang X, Miller W. A Time-Efficient, Linear-Space Local Similarity Algorithm. Advances in Applied Mathematics. 1991;12:337-57.

[97] Rice P, Longden I, Bleasby A. EMBOSS: The European Molecular Biology Open Software Suite. Trends in Genetics. 2000;16:276-7.

[98] Sievers F, Wilm A, Dineen D, Gibson TJ, Karplus K, Li W, et al. Fast, scalable generation of highquality protein multiple sequence alignments using Clustal Omega. Molecular systems biology. 2011;7:539.

[99] Sievers F, Higgins DG. Clustal Omega for making accurate alignments of many protein sequences. Protein Science. 2018;27:135-45.

[100] Crooks GE, Hon G, Chandonia J-M, Brenner SE. WebLogo: a sequence logo generator. Genome Research. 2004;14:1188-90.

[101] Konopka BM, Marciniak M, Dyrka W. Quantiprot - a Python package for quantitative analysis of protein sequences. BMC bioinformatics. 2017;18:339.

[102] Piovesan D, Tabaro F, Mičetić I, Necci M, Quaglia F, Oldfield CJ, et al. DisProt 7.0: a major update of the database of disordered proteins. Nucleic Acids Research. 2017;45:D219-D27.

[103] Rosteck PR, Reynolds PA, Hershberger CL. Homology between proteins controlling

Streptomyces fradiae tylosin resistance and ATP-binding transport. Gene. 1991;102:27-32.

[104] Murzin AG. Structural principles for the propeller assembly of beta-sheets: the preference for seven-fold symmetry. Proteins. 1992;14:191-201.

[105] Lamb JR, Tugendreich S, Hieter P. Tetratrico peptide repeat interactions: to TPR or not to TPR? Trends in Biochemical Sciences. 1995;20:257-9.

[106] Aravind L, Koonin EV. Classification of the caspase-hemoglobinase fold: detection of new families and implications for the origin of the eukaryotic separins. Proteins. 2002;46:355-67.

[107] Mushegian AR, Koonin EV. Unexpected sequence similarity between nucleosidases and phosphoribosyltransferases of different specificity. Protein Science: A Publication of the Protein Society. 1994;3:1081-8.

[108] Bonnert TP, Garka KE, Parnet P, Sonoda G, Testa JR, Sims JE. The cloning and characterization of human MyD88: a member of an IL-1 receptor related family. FEBS letters. 1997;402:81-4.

[109] Balguerie A, Dos Reis S, Coulary-Salin B, Chaignepain S, Sabourin M, Schmitter JM, et al. The sequences appended to the amyloid core region of the HET-s prion protein determine higher-order aggregate organization in vivo. J Cell Sci. 2004;117:2599-610.

[110] Bergès T, Barreau C. Heat-shock at an elevated temperature improves transformation efficiency of protoplats from Podospora anserina. J Gen Microbiol. 1989;135:601-4.

[111] Dos Reis S, Coulary-Salin B, Forge V, Lascu I, Begueret J, Saupe SJ. The HET-s prion protein of the filamentous fungus Podospora anserina aggregates in vitro into amyloid-like fibrils. J Biol Chem. 2002;277:5703-6. 
Table 1. Phylogenetic distribution of Bell domains and NLRs in bacteria

\begin{tabular}{|c|c|c|c|c|}
\hline phylum & \#genomes & NBARC-TPR & NACHT-WD & Bell \\
\hline d__Bacteria;p_Acidobacteriota & 91 & 9 & 0 & 2 \\
\hline d__Bacteria;p_Actinobacteriota & 13236 & 2533 & 284 & 930 \\
\hline d__Bacteria;p__Bacteroidota & 2858 & 38 & 8 & 30 \\
\hline d__Bacteria;p__Campylobacterota & 3368 & 3 & 0 & 0 \\
\hline d__Bacteria;p__Chloroflexota & 273 & 48 & 9 & 12 \\
\hline d__Bacteria;p__Cyanobacteriota & 513 & 223 & 152 & 173 \\
\hline d__Bacteria;p__Deinococcota & 81 & 6 & 0 & 0 \\
\hline d__Bacteria;p__Desulfobacterota & 267 & 6 & 1 & 3 \\
\hline d__Bacteria;p__Firmicutes & 32747 & 386 & 2 & 0 \\
\hline d__Bacteria;p__Firmicutes_A & 3076 & 34 & 0 & 0 \\
\hline d__Bacteria;p__Firmicutes_B & 130 & 3 & 0 & 0 \\
\hline d__Bacteria;p_Firmicutes_C & 182 & 1 & 0 & 0 \\
\hline d__Bacteria;p__Fusobacteriota & 195 & 0 & 0 & 0 \\
\hline d__Bacteria;p__Myxococcota & 91 & 20 & 18 & 0 \\
\hline d__Bacteria;p__Omnitrophota & 88 & 0 & 0 & 0 \\
\hline d__Bacteria;p_Patescibacteria & 1772 & 10 & 0 & 0 \\
\hline d__Bacteria;p__Planctomycetota & 178 & 4 & 0 & 0 \\
\hline d__Bacteria;p_Proteobacteria & 51737 & 146 & 26 & 83 \\
\hline d__Bacteria;p__Spirochaetota & 808 & 4 & 0 & 0 \\
\hline d__Bacteria; $p_{\text {___Verrucomicrobiota }}$ & 582 & 8 & 0 & 0 \\
\hline
\end{tabular}


Table 2. Phylogenetic distribution of Bell-domains and NLRs in Actinobacteria and Cyanobacteria

\begin{tabular}{|c|c|c|c|c|}
\hline family & \#genomes & $\begin{array}{c}\text { NBARC- } \\
\text { TPR }\end{array}$ & $\begin{array}{c}\text { NACHT- } \\
\text { WD }\end{array}$ & Bell \\
\hline c__Actinobacteria & 12979 & 2523 & 282 & 927 \\
\hline o__Actinomycetales;f__Actinomycetaceae & 201 & 22 & 6 & 0 \\
\hline o__Actinomycetales;f__Bifidobacteriaceae & 558 & 0 & 0 & 0 \\
\hline o__Actinomycetales;f__Brevibacteriaceae & 45 & 2 & 0 & 0 \\
\hline o__Actinomycetales;f__Cellulomonadaceae & 75 & 15 & 0 & 1 \\
\hline o__Actinomycetales;f__Demequinaceae & 32 & 0 & 0 & 0 \\
\hline o__Actinomycetales;f__Dermabacteraceae & 26 & 0 & 0 & 0 \\
\hline o__Actinomycetales;f___Dermatophilaceae & 79 & 25 & 2 & 2 \\
\hline o__Actinomycetales;f__Microbacteriaceae & 415 & 32 & 0 & 4 \\
\hline o__Actinomycetales;f__Micrococcaceae & 308 & 14 & 8 & 5 \\
\hline o__Corynebacteriales;f__Corynebacteriaceae & 9174 & 789 & 20 & 185 \\
\hline o__Corynebacteriales;f__Geodermatophilaceae & 40 & 13 & 0 & 2 \\
\hline o__Corynebacteriales;f__Micromonosporaceae & 202 & 202 & 36 & 53 \\
\hline o__Corynebacteriales;f__Pseudonocardiaceae & 198 & 169 & 20 & 91 \\
\hline o__Frankiales;f__Frankiaceae & 40 & 38 & 20 & 9 \\
\hline o__Jiangellales;f__Jiangellaceae & 7 & 7 & 0 & 0 \\
\hline o__Nanopelagicales;f__Nanopelagicaceae & 31 & 0 & 0 & 0 \\
\hline o__Propionibacteriales;f__Nocardioidaceae & 66 & 24 & 0 & 0 \\
\hline o__Propionibacteriales;f__Propionibacteriaceae & 287 & 3 & 0 & 0 \\
\hline o__Streptomycetales;f__Streptomycetaceae & 1049 & 1047 & 130 & 540 \\
\hline o__Streptosporangiales; f__Streptosporangiaceae & 100 & 99 & 37 & 33 \\
\hline c_Cyanobacteriia & 467 & 223 & 152 & 173 \\
\hline o__Cyanobacteriales;f__Coleofasciculaceae & 7 & 7 & 6 & 6 \\
\hline o__Cyanobacteriales;f_Cyanobacteriaceae & 21 & 2 & 1 & 3 \\
\hline o__Cyanobacteriales;f__Microcystaceae & 54 & 39 & 6 & 37 \\
\hline o__Cyanobacteriales;f__Nostocaceae & 127 & 99 & 84 & 86 \\
\hline o__Cyanobacteriales;f__Phormidiaceae & 32 & 26 & 11 & 9 \\
\hline o__Eurycoccales;f__Leptococcaceae & 8 & 0 & 0 & 0 \\
\hline o__Leptolyngbyales;f__Leptolyngbyaceae & 10 & 9 & 4 & 9 \\
\hline o__Phormidesmiales;f__Phormidesmiaceae & 9 & 8 & 7 & 4 \\
\hline o__Pseudanabaenales;f__Pseudanabaenaceae & 8 & 3 & 3 & 2 \\
\hline o__Synechococcales_A;f__Cyanobiaceae & 133 & 0 & 1 & 0 \\
\hline
\end{tabular}


Table 3. Sequences selected for functional studies

\begin{tabular}{lll}
\hline Motif/Domain/Strain & $\begin{array}{l}\text { Accession number and coordinates } \\
\text { of } \text { in vivo/in vitro } \text { constructs }\end{array}$ & Code name \\
\hline BASS1/Bell/Streptomyces coelicolor A3(2) & CAB66307.1 (110-139)/(110-139), (38-139) & B1-B S.c. \\
BASS1/NLR/Streptomyces coelicolor A3(2) & CAB66306.1 (1-34)/- & B1-N S.c \\
BASS3/Bell/Streptomyces atratus & WP_037701008.1 (70-124)/(70-124), (97-124) & B3-B S.a. \\
BASS3/NLR/Streptomyces atratus & WP_037701012.1 (1-37)/- & B3-N S.a. \\
BASS3/Bell/Nostoc punctiforme PCC 73102 & ACC79696.1 $(94-126) /(94-126)$ & B3-B $N . p$. \\
BASS3/NLR/Nostoc punctiforme PCC 73102 & ACC79697.1 $(1-38) /-$ & B3-N N.p. \\
BASS3/Bell/Nocardia fusca & WP_063130184.1 (74-128)/(74-128), (100-128) & B3-B $N . f$. \\
\hline
\end{tabular}


Table 4. Spontaneous prion formation rates of selected bacterial motifs

\begin{tabular}{|c|c|c|c|c|c|c|c|c|c|c|c|c|}
\hline \multirow[t]{3}{*}{ transgene } & \multicolumn{12}{|c|}{ time after transfection in days } \\
\hline & \multicolumn{3}{|c|}{$5 \mathrm{~d}$. } & \multicolumn{3}{|c|}{$11 \mathrm{~d}$} & \multicolumn{3}{|c|}{$19 \mathrm{~d}}$. & \multicolumn{3}{|c|}{$26 \mathrm{~d}$. } \\
\hline & {$[\mathrm{b} *]$} & [b] & $\%$ & {$\left[b^{*}\right]$} & [b] & $\%$ & {$\left[b^{*}\right]$} & [b] & $\%$ & {$\left[b^{*}\right]$} & [b] & $\%$ \\
\hline B1-B S.c. (RFP) & 30 & 19 & 39 & 7 & 30 & 81 & 3 & 36 & 92 & 3 & 37 & 93 \\
\hline B1-B S.c. (GFP) & 31 & 5 & 14 & 11 & 14 & 56 & 8 & 17 & 68 & 0 & 18 & 100 \\
\hline B1-N S.c. (RFP) & 32 & 21 & 39 & 9 & 29 & 76 & 8 & 33 & 81 & 3 & 34 & 92 \\
\hline B1-N S.c. (GFP) & 15 & 17 & 53 & 7 & 19 & 73 & 5 & 26 & 84 & 0 & 29 & 100 \\
\hline B3-B N.p. (RFP) & 16 & 1 & 6 & 5 & 3 & 38 & 4 & 4 & 50 & 3 & 10 & 77 \\
\hline B3-B N.p. (GFP) & 33 & 2 & 6 & 12 & 10 & 46 & 8 & 14 & 64 & 1 & 17 & 94 \\
\hline B3-N N.p. (RFP) & 30 & 7 & 19 & 6 & 11 & 65 & 6 & 16 & 73 & 3 & 17 & 85 \\
\hline B3-N N.p. (GFP) & 38 & 8 & 17 & 20 & 8 & 29 & 8 & 12 & 60 & 0 & 15 & 100 \\
\hline B3-B S.a. (RFP) & 33 & 4 & 11 & 14 & 16 & 53 & 8 & 22 & 73 & 3 & 25 & 89 \\
\hline B3-B S.a. (GFP) & 5 & 171 & 97 & 1 & 175 & 99 & nd & nd & nd & 0 & 83 & 100 \\
\hline B3-N S.a. (RFP) & 28 & 12 & 30 & 8 & 16 & 67 & 7 & 17 & 71 & 3 & 17 & 86 \\
\hline B3-N S.a. (GFP) & 16 & 33 & 67 & 7 & 35 & 83 & 6 & 36 & 86 & 1 & 38 & 97 \\
\hline B3-B N.f. (RFP) & 44 & 8 & 15 & 13 & 9 & 41 & 8 & 17 & 68 & 1 & 11 & 92 \\
\hline B3-B N.f. (GFP) & 6 & 92 & 94 & 0 & 98 & 100 & nd & nd & nd & nd & nd & nd \\
\hline
\end{tabular}

nd, not determined. , [ $\left.\mathrm{b}^{*}\right]$ or $[\mathrm{b}]$, number of strains in the given phenotypic state, \%, percentage of strains in the [b] prion state 
Journal Pre-proofs

Table 5. Effect of proline mutations of the BASS3 motif from S. atratus (B3-B S.a.) on prion formation in $P$. anserina

\begin{tabular}{|c|c|c|c|c|c|c|c|c|c|c|c|}
\hline & & & tane & Is pri & forma & ion & & & \multirow{2}{*}{\multicolumn{3}{|c|}{$\begin{array}{l}\text { Induced prion } \\
\text { formation }\end{array}$}} \\
\hline \multicolumn{9}{|c|}{ Days after transfection } & & & \\
\hline \multicolumn{3}{|c|}{4 days } & \multicolumn{3}{|c|}{8 days } & \multicolumn{3}{|c|}{15 days } & & & \\
\hline$\left[b^{*}\right]$ & [b] & & {$\left[b^{*}\right]$} & [b] & & {$\left[b^{*}\right]$} & [b] & & {$\left[b^{*}\right]$} & [b] & \\
\hline $\mathrm{n}$ & $\mathrm{n}$ & $\%$ & $\mathrm{n}$ & $\mathrm{n}$ & $\%$ & $\mathrm{n}$ & $\mathrm{n}$ & $\%$ & $\mathrm{n}$ & $\mathrm{n}$ & $\%$ \\
\hline 4 & 46 & 92.0 & 1 & 49 & 98.0 & 0 & 50 & 100 & 0 & 24 & 100 \\
\hline 37 & 0 & 0 & 37 & 0 & 0 & 37 & 0 & 0 & 24 & 0 & 0 \\
\hline 49 & 0 & 0 & 49 & 0 & 0 & 49 & 0 & 0 & 24 & 0 & 0 \\
\hline 24 & 0 & 0 & 24 & 0 & 0 & 24 & 0 & 0 & 24 & 0 & 0 \\
\hline 32 & 0 & 0 & 32 & 0 & 0 & 32 & 0 & 0 & 24 & 0 & 0 \\
\hline
\end{tabular}




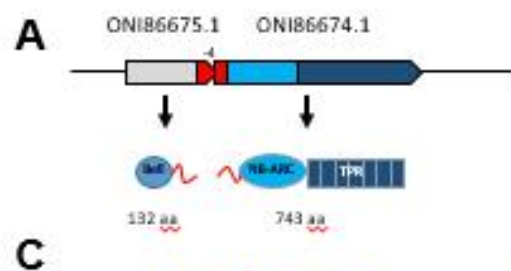
B
ONIB6675.1 (104-128) YTVDÄREAQGVQIGEHNSMTLNPG
ONI86674.1 (11-34) PAVDARYGQGIQIGDHNTQHVHLT
RIP3 (448-471) PLVNIYNCSGVQVGDNIYLTMQQT
RIP1 (483-506) IKYTIYNSTGIQIGAYNYMEIGGT

C

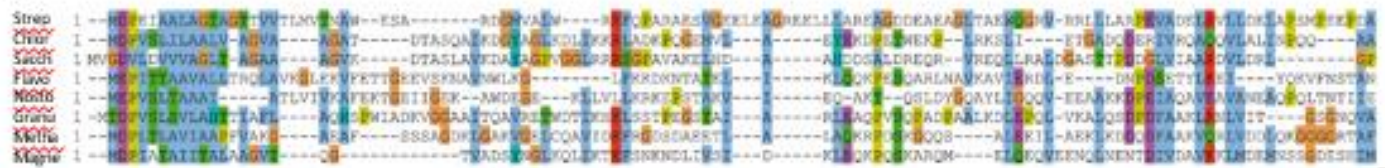

D

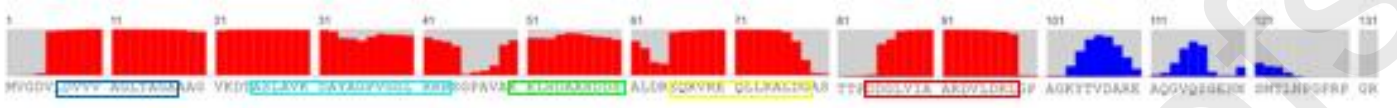

E

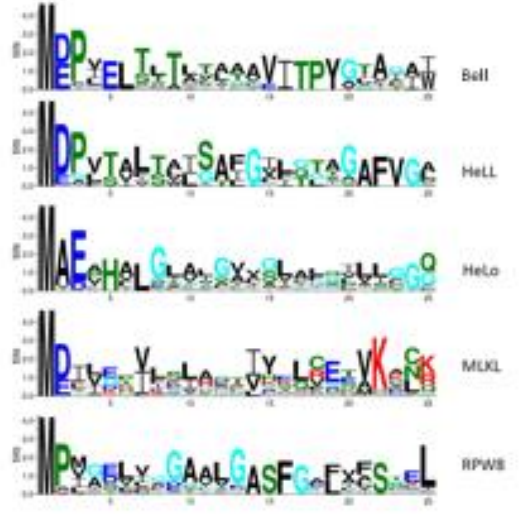

$\mathbf{F}$

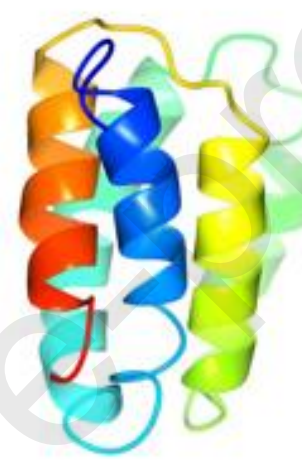




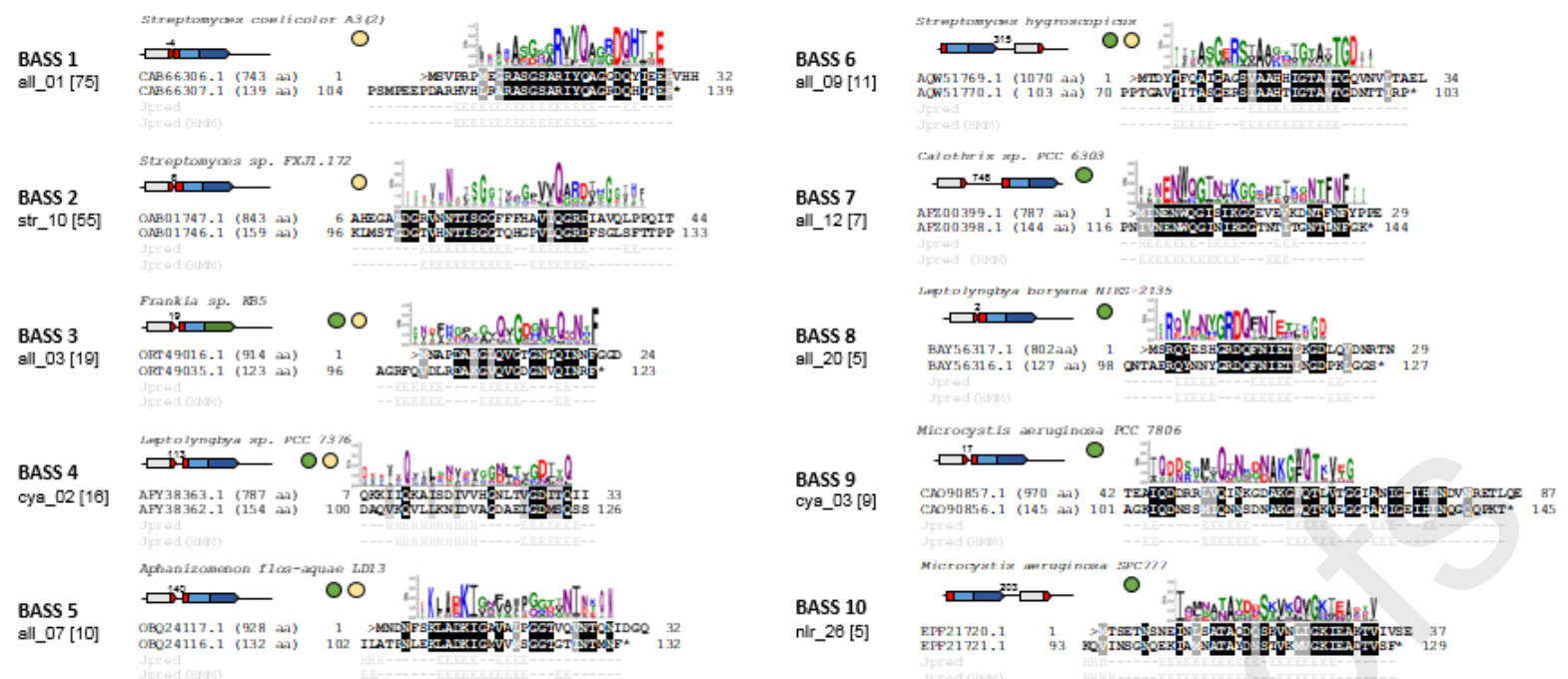




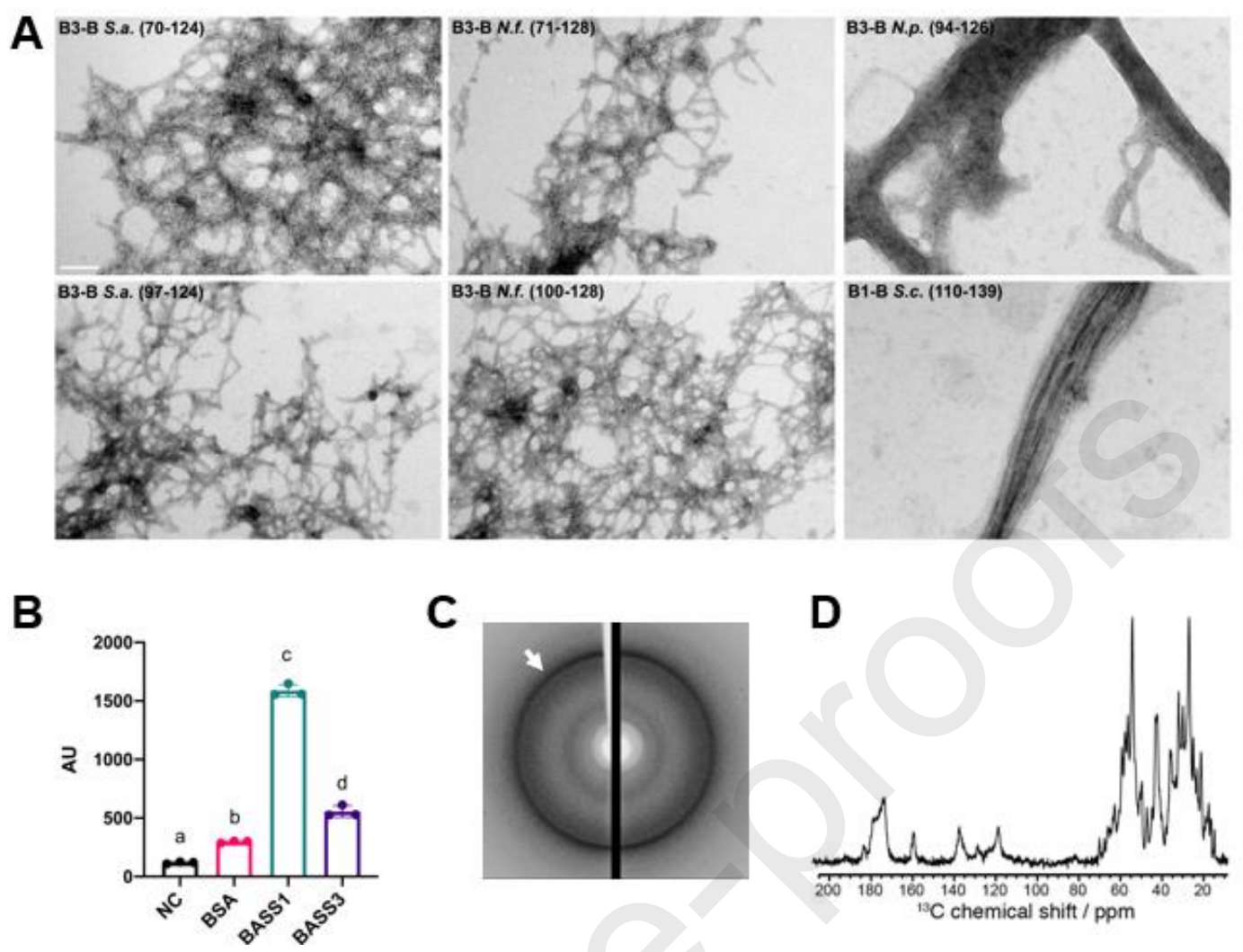




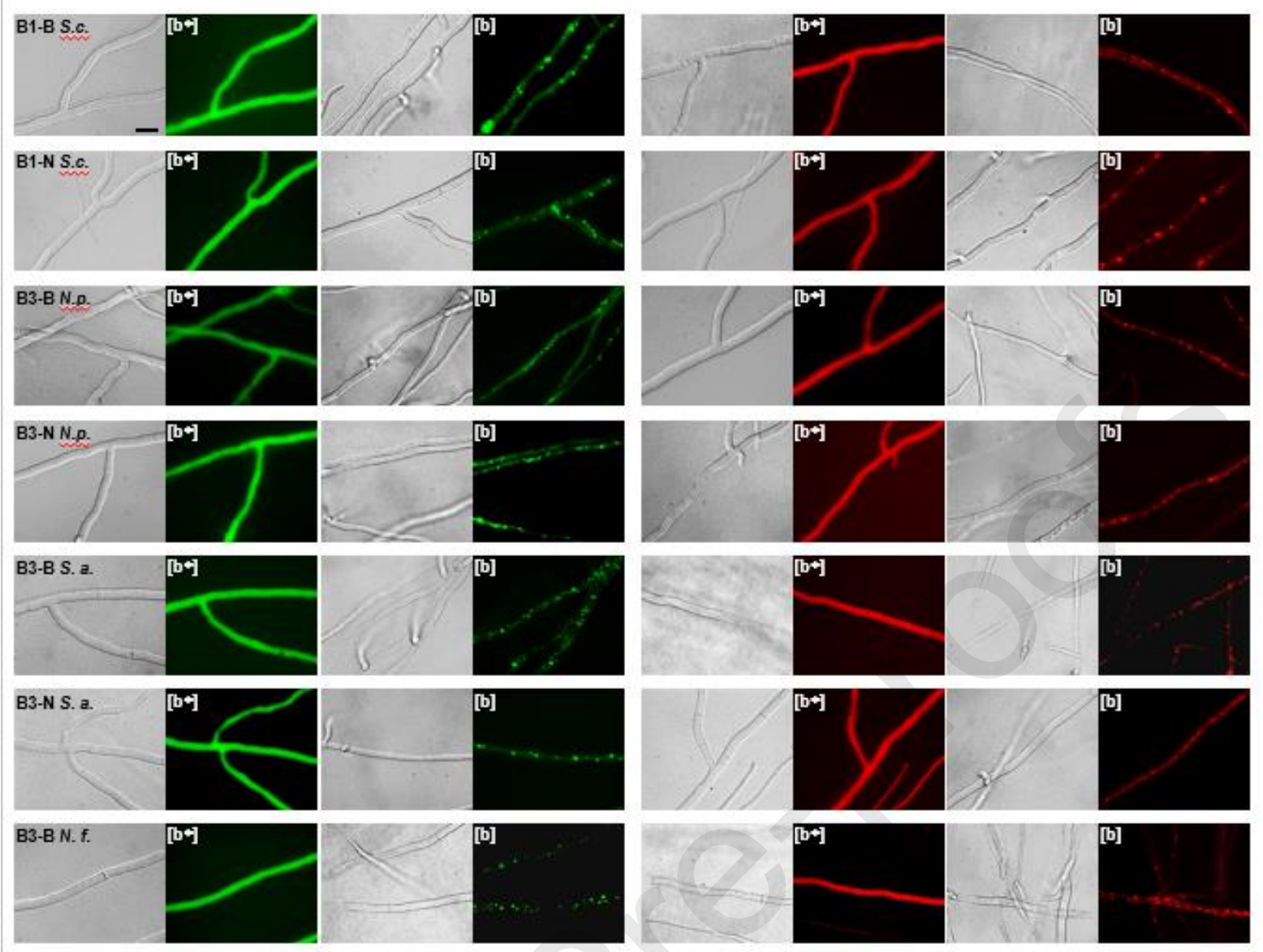


A

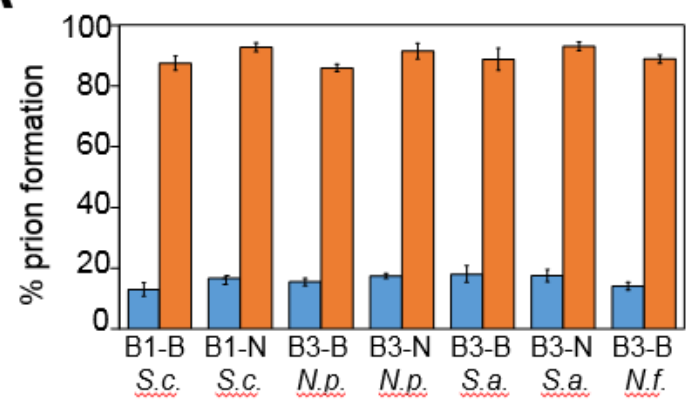

B

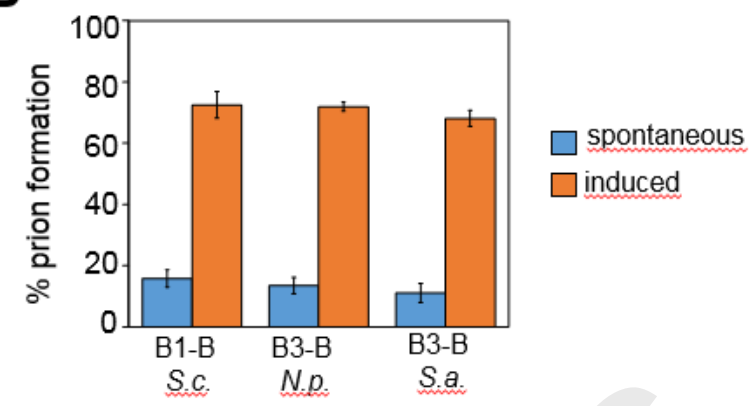




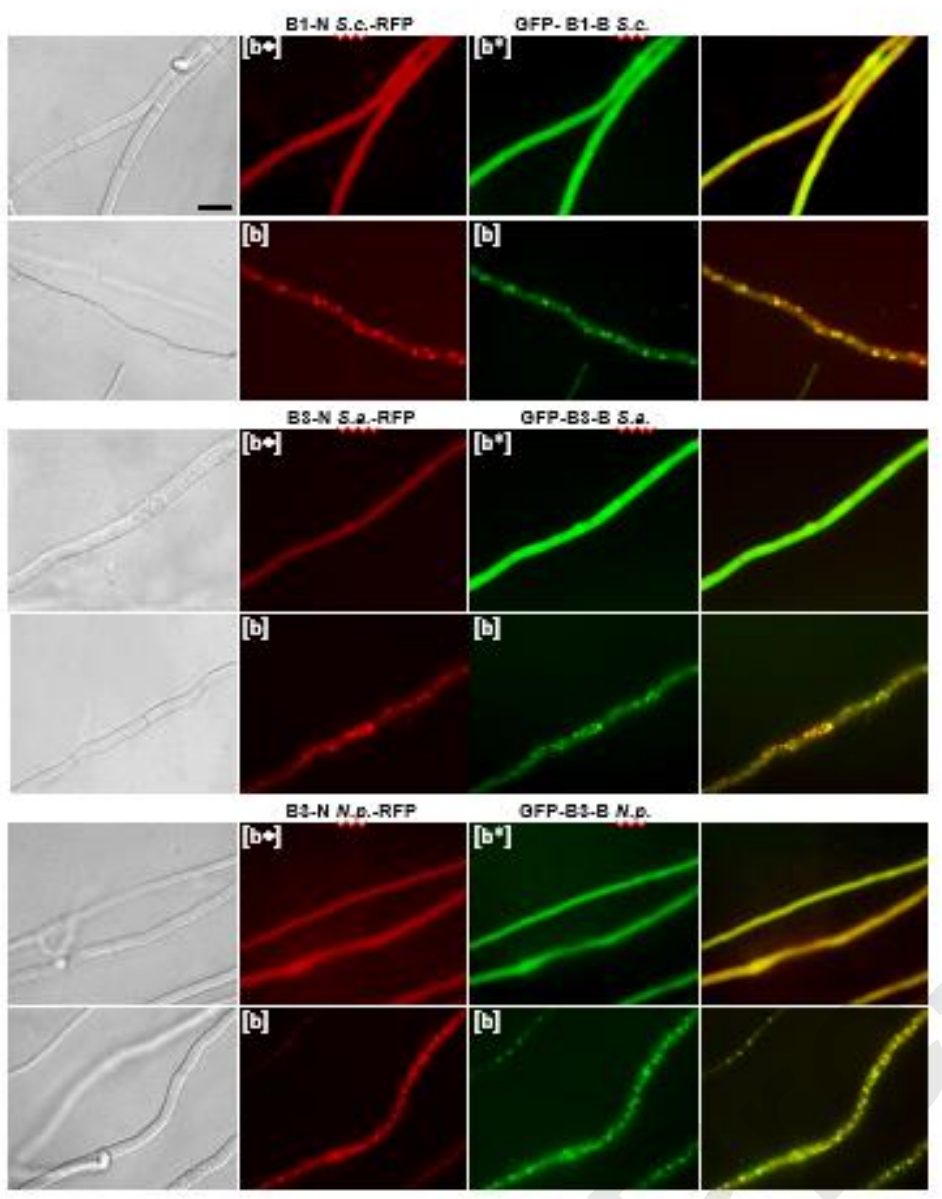




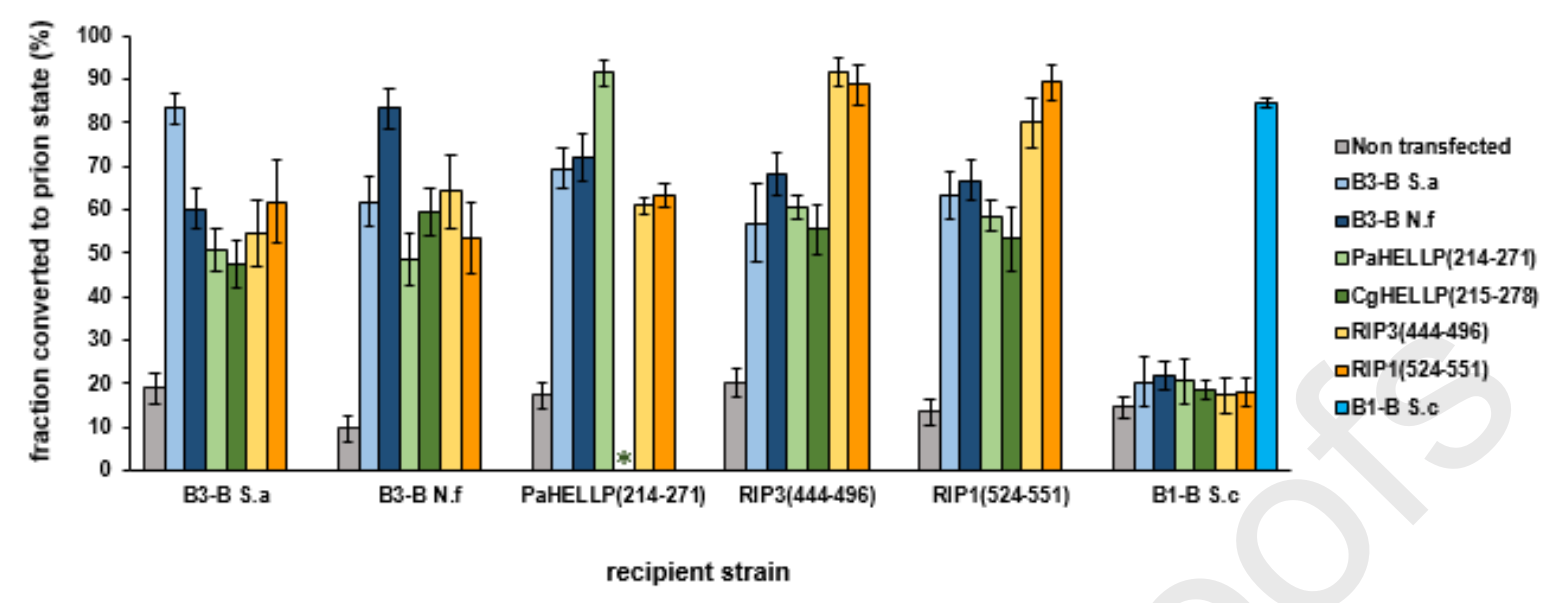


Graphical abstract
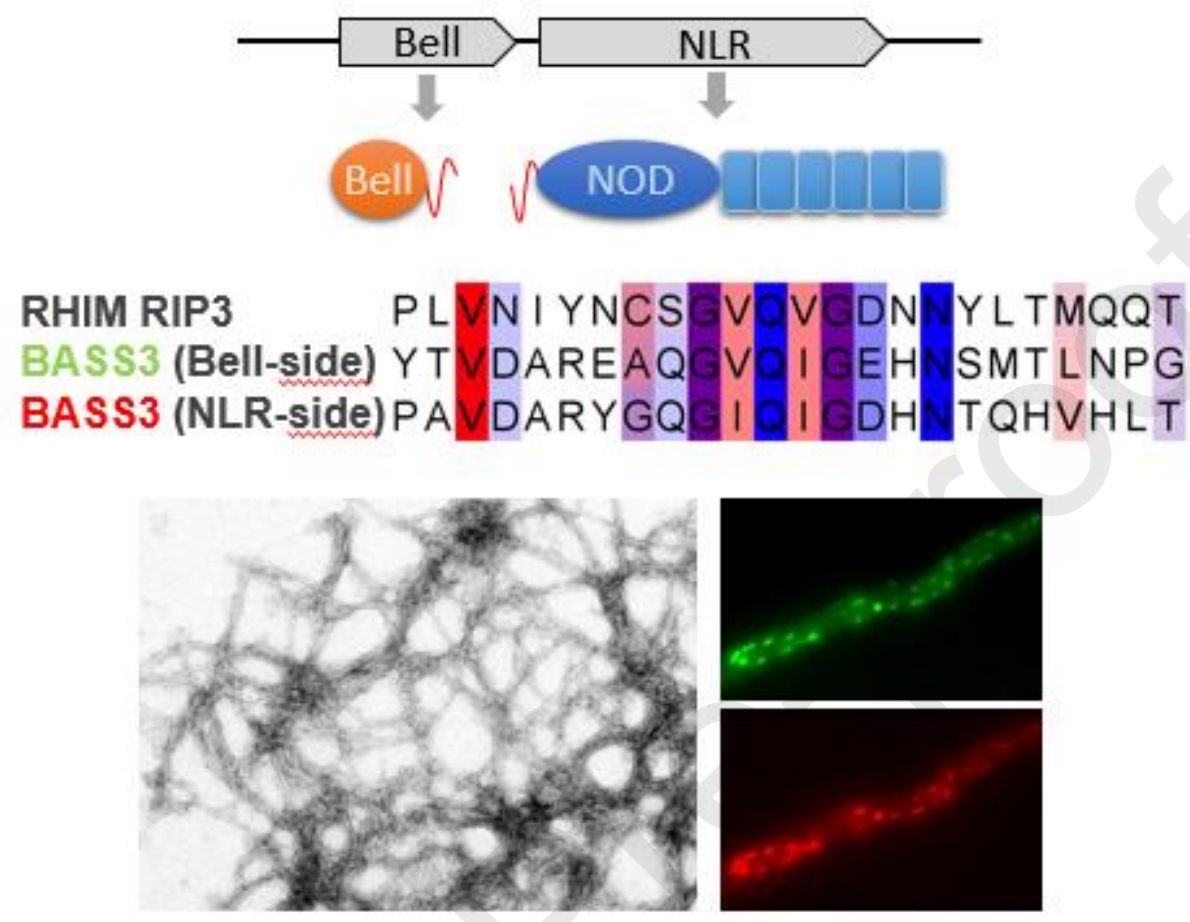
Highlights

- Bacterial genomes contain amyloid motifs related to known amyloid signaling motifs from fungi and animals and associated to homologs of Nod-like receptors

- BASS motifs occur mainly in multicellular bacteria

- Selected BASS-motifs form amyloids in vitro and propagate as prions in vivo in a fungal model

- BASS3 motifs cross-seed in vivo with RHIM and fungal PP-motifs 
Witold Dyrka: conceptualization, methodology, investigation, data curation, visualization, writing, funding acquisition, Virginie Coustou: methodology, investigation, visualization, writing, Asen Daskalov: conceptualization, investigation, Alons Lends: investigation, Thierry Bardin: investigation, Mélanie Berbon: investigation Brice Kauffmann : investigation, methodology, Corinne Blancard: investigation, Bénédicte Salin : investigation, Antoine Loquet: conceptualization, methodology, writing, funding acquisition, Sven J. Saupe : project administration, funding acquisition, writing, conceptualization, investigation, vizualization 
Declaration of interests

$\bigotimes$ The authors declare that they have no known competing financial interests or personal relationships that could have appeared to influence the work reported in this paper.

$\square$ The authors declare the following financial interests/personal relationships which may be considered as potential competing interests: 